\title{
Commuting Signatures and Verifiable Encryption
}

\author{
Georg Fuchsbauer* \\ Dept. Computer Science, University of Bristol, UK \\ georg@cs.bris.ac.uk
}

\begin{abstract}
Verifiable encryption allows one to encrypt a signature while preserving its public verifiability. We introduce a new primitive called commuting signatures and verifiable encryption that extends this in multiple ways, such as enabling encryption of both signature and message while proving validity. More importantly, given a ciphertext, a signer can create a verifiably encrypted signature on the encrypted (unknown) message, which leads to the same result as first signing the message and then verifiably encrypting the message/signature pair; thus, signing and encrypting commute. Our instantiation is based on the recently introduced automorphic signatures and Groth-Sahai proofs, which we show to be homomorphic. We also prove a series of other properties and provide a novel approach to simulation.

As an application, we give an instantiation of delegatable anonymous credentials, a primitive introduced by Belenkiy et al. Our construction is arguably simpler than theirs and it is the first to provide non-interactive (and thus concurrently secure) issuing and delegation protocols, which are significantly more efficient. Moreover, the size of our credentials and the cost of verification are less than half of those of the previous instantiation. All our constructions are proven secure in the standard model under known non-interactive assumptions.
\end{abstract}

Keywords: Verifiably encrypted signatures, blind signatures, anonymous credentials, Groth-Sahai proofs.

\section{Introduction}

Verifiably encrypted signatures let us sign a message, encrypt the signature, and make a proof asserting that the ciphertext contains a valid signature. Suppose the message is only available as an encryption. We cannot make a signature on the plaintext, as this would contradict the security of the encryption scheme 1 . But could we instead, given a ciphertext, produce a verifiable encryption of a signature on the plaintext?

We show that such a functionality is feasible and moreover give a practical instantiation of it. We then use this new primitive to build the first non-interactively delegatable anonymous credential scheme: given an encrypted public key, a delegator can make a verifiably encrypted certificate for the key, which acts as a credential.

\footnotetext{
* Work done while at École normale supérieure, Paris, France. The author has been supported by the French ANR 07-TCOM-013-04 PACE Project, the European Commission through the ICT Program under Contract ICT-2007-216676 ECRYPT II and EPSRC Grant EP/H043454/1.

${ }^{1}$ Given two messages and the encryption of one of them, a signature on the plaintext could be used to decide which one was encrypted.
} 
Delegatable Anonymous Credentials. Access control that respects users' privacy concerns is a challenging problem in security. To gain access to resources, a participant must prove to possess the required credential issued by an authority. To increase manageability of the system, the authority usually does not issue credentials directly to each user, but relies on intermediate layers in the hierarchy. For example, a system administrator issues credentials to webmasters for using his server; the latter may then create forums and delegate rights to moderators, who can give posting privileges to users.

Web-based social network services are enjoying a huge popularity and represent another area of application for credentials. Registered users can be given credentials to access services, which they delegate to introduce and recommend friends and friend of friends. The recent rise of concern about protection of privacy in such networks motivates anonymous credentials: a user can obtain a credential and prove possession of it without revealing neither her identity nor that of the user who delegated it to her.

In practice, (non-anonymous) delegation of rights is usually realized by certifying (i.e., signing) the public key of the delegated user. Consecutive delegation leads to a certification chain, consisting of public keys and certificates linking them, starting with the original issuer of the credential. A user in the chain can delegate the credential by signing the delegatee's public key and appending the certificate to the credential.

Anonymous credentials [Cha85, Dam90, Bra99, LRSW00, CL01, CL04, BCKL08] aim to provide a functionality similar to certificates without revealing information about the user's identity when obtaining or showing a credential. However, the goal of reconciling delegatability and anonymity remained elusive - until recently. Chase and Lysyanskaya [CL06] show theoretical feasibility of delegatable anonymous credentials, but their size is exponential in the number of delegations. A breakthrough was then made in $\left[\mathrm{BCC}^{+} 09\right]$, where Belenkiy et al. (BCCKLS) introduce a new approach using a noninteractive zero-knowledge (NIZK) proof system [BFM88] with randomizable proofs: anyone can transform such a proof into a new proof of the same statement that cannot be linked to the original one. A credential is a proof of knowledge of a certification chain that can be randomized before being delegated or shown, which guarantees anonymity and unlinkability.

In their model, each user holds a secret key which can be used to produce multiple unlinkable pseudonyms $N y m$. A user $A$ can be known to user $O$ as $N y m_{A}^{(O)}$ and to $B$ as $N y m_{A}^{(B)}$. Given a credential issued by $O$ for $N y m_{A}^{(O)}, A$ can transform it into a credential from $O$ for $N y m_{A}^{(B)}$ and show it to $B$. Moreover, $A$ can delegate the credential to user $C$, known to $A$ as $N y m_{C}^{(A)}$. $C$ can then show a credential from $O$ for $N y m_{C}^{(D)}$ to user $D$ (without revealing neither $N y m_{A}^{(C)}$ nor $N y m_{C}^{(A)}$ ), or redelegate it. Delegation preserves anonymity, in that delegator and delegatee learn nothing more about each other than their respective pseudonyms. This is formalized by requiring that there exist a simulator that can produce pseudonyms and credentials for them without knowing any secrets.

In the instantiation of $\left[\mathrm{BCC}^{+} 09\right]$, delegation is fairly complex and interactive-in contrast to non-anonymous credentials, where it suffices to know a user's public key in order to issue or delegate a credential to her. We bridge this gap by giving an instantiation that enables non-interactive delegation: given a pseudonym Nym, a delegator can produce a ready credential for the holder of Nym without any interaction. Note that a non-interactive delegation protocol immediately yields security against 
concurrent attacks, where an adversary might simultaneously run protocols for delegating and being delegated credentials with honest users. This was not considered in the BCCKLS model.

Commuting Signatures. Our main building block for non-interactively delegatable anonymous credentials is a new primitive we call commuting signature and verifiable encryption (or commuting signature for short), which we sketch in the following and formally define in Section 3. It combines a digital signature scheme, an encryption scheme and a proof system with the following properties: given a verification key $v k$, a message $M$ and a signature $\Sigma$ on $M$ under $v k$, we can encrypt any subset of $\{v k, M, \Sigma\}$ and add a proof (which leaks no more information) that the plaintexts are a key, a message and a valid signature-which makes the encryptions verifiable.

For consistency with the Groth-Sahai methodology [GS08], we also say commitment instead of encryption, as their commitments to group elements, which we will use, are extractable, i.e., we can recover the committed value (and thus "decrypt") using an extraction key. An extractable commitment to a signature together with a proof of validity is a proof of knowledge (PoK) of a signature, and at the same time a verifiably encrypted signature (VES) [BGLS03, RS09].

We denote committing to signatures by Com, committing to messages by $\mathrm{Com}_{\mathcal{M}}$, and proving validity by Prove. A proof for a committed signature is denoted by $\tilde{\pi}$, and for a committed message by $\bar{\pi}$. If both are committed, we write $\pi$, and if the verification key is committed too, we write $\widehat{\pi}$ (" $\sim$ " for signature and " $\wedge$ " for $\underline{v} k$ ). Besides allowing us to prove validity of committed values, a commuting signature scheme provides the following functionalities, neither of which requires knowledge of the extraction key:

SigCom. Given a commitment $\mathbf{C}_{M}$ to a message $M$ and a signing key sk, SigCom produces a commitment $\mathbf{c}_{\Sigma}$ to a signature $\Sigma$ on $M$ under $s k$, and a proof $\pi$ that the content of $\mathbf{c}_{\Sigma}$ is a valid signature on the content of $\mathbf{C}_{M}$.

$\operatorname{AdC}_{\mathcal{S}}$. Given a commitment $\mathbf{C}_{M}$ to $M$, a signature $\Sigma$ on $M$ and a proof $\bar{\pi}$ of validity of $\Sigma$ on the content of $\mathbf{C}_{M}$, we can make a commitment $\mathbf{c}_{\Sigma}$ to $\Sigma$ using randomness $\rho_{\Sigma}$. Then $\operatorname{AdC}_{\mathcal{S}}$ ("adapt when committing to signature") allows us to adapt $\bar{\pi}$ to a proof for $\mathbf{C}_{M}$ and $\mathbf{c}_{\Sigma}$ : given $\left(\mathbf{C}_{M}, \Sigma, \rho_{\Sigma}, \bar{\pi}\right)$ it returns a proof $\pi$ that the content of $\mathbf{c}_{\Sigma}$ is a valid signature on the content of $\mathbf{C}_{M}$. $\operatorname{AdD}_{\mathcal{S}}$ ("adapt proof when $d e$ committing") does the converse: given a committed message $\mathbf{C}_{M}$, a committed signature $\mathbf{c}_{\Sigma}$ together with the used randomness $\rho_{\Sigma}$, and a proof $\pi$ for $\mathbf{C}_{M}$ and $\mathbf{c}_{\Sigma}$, $\operatorname{AdD}_{\mathcal{S}}$ outputs a proof $\bar{\pi}$ of validity of the signature $\Sigma$ on the committed message.

$\mathrm{AdC}_{\mathcal{M}}$. Analogously we define proof adaptation for the message. Given $M$, a commitment $\mathbf{c}_{\Sigma}$ to a signature on $M$ and a proof of validity $\widetilde{\pi}, \operatorname{AdC}_{\mathcal{M}}$ transforms the proof to the case when the message is committed as well. $\mathrm{AdD}_{\mathcal{M}}$ is given commitments $\mathbf{C}_{M}$ to a message $M$ and $\mathbf{c}_{\Sigma}$ to a signature, the randomness $\rho_{M}$ for $\mathbf{C}_{M}$ and a proof $\pi$. It adapts $\pi$ to a proof $\tilde{\pi}$ that the content of $\mathbf{c}_{\Sigma}$ is a valid signature on $M$.

$\mathrm{AdC}_{\mathcal{K}}$. We can also adapt proofs when (de)committing to the verification key. Given commitments $\mathbf{C}_{M}$ and $\mathbf{c}_{\Sigma}$, a proof of validity $\pi$ w.r.t. a verification key $v k$, and

\footnotetext{
${ }^{2}$ While for VES, encryption suffices to be one-way (opacity means it is hard to extract a signature), we require verifiable encryptions of different signatures to be indistinguishable.
} 


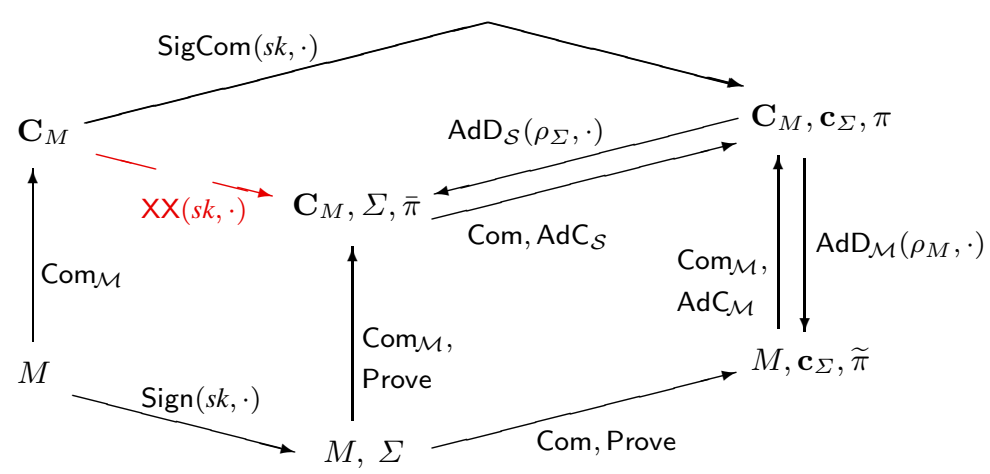

Fig. 1. Diagram representing a system of commuting signatures and verifiable encryption

randomness $\rho_{v k}, \mathrm{AdC}_{\mathcal{K}}$ outputs a proof $\widehat{\pi}$ that the content of $\mathbf{c}_{\Sigma}$ is a signature on the content of $\mathbf{C}_{M}$ valid under $v k$ given as a commitment $\mathbf{c}_{v k}$ with randomness $\rho_{v k} . \operatorname{AdD}_{\mathcal{K}}$ is given $\left(v k, \rho_{v k}, \mathbf{C}_{M}, \mathbf{c}_{\Sigma}, \widehat{\pi}\right)$ and adapts the proof $\widehat{\pi}$ for $\left(\mathbf{c}_{v k}, \mathbf{C}_{M}, \mathbf{c}_{\Sigma}\right)$, where $\mathbf{c}_{v k}$ commits to $v k$ with randomness $\rho_{v k}$, to a proof for $\left(v k, \mathbf{C}_{M}, \mathbf{c}_{\Sigma}\right)$.

We require that committing, signing and the above functionalities all commute with each other, that is, it does not matter in which order they are executed; e.g., signing a message, committing to the message and the signature, and proving validity yields the same as committing to the message and then running SigCom. Thus, the diagram in Figure 1 commutes. Note that due to the argument given in Footnote 1 there cannot exist a functionality $\mathrm{XX}$ that is given a commitment $\mathbf{C}_{M}$ to a message $M$ and a secret key $s k$, and outputs a signature $\Sigma$ on $M$.

Besides verifiably encrypted signatures, commuting signatures imply blind signatures, and moreover CL signatures [CL02] and P-signatures [BCKL08], both building blocks for protocols providing privacy. They let a user obtain a signature on a committed value from a signer by running an issuing protocol. The user can then make a proof of knowledge of that signature, which is verifiable given the commitment. SigCom provides a non-interactive issuing, which directly gives the user a (randomizable) proof of knowledge of such a signature (see the full version [Fuc10]).

Instantiating Commuting Signatures. Blind signatures [Cha83, PS96] enable a user to obtain a signature on a message in a way that the signer cannot link the resulting message/signature pair to its issuing. In [Fuc09, $\left.\mathrm{AFG}^{+} 10\right]$ the author gives an efficient implementation with round-optimal issuing [Fis06], where after sending information to the signer, the user can immediately derive the blind signature from the signer's response. In this scheme, the user randomizes the message, makes (extractable) commitments to the message and the randomness, and adds a witness-indistinguishable (WI) proof that the commitments contain the correct values.

The user sends these values to the signer, who learns nothing about the message from them. The signer fabricates a "pre-signature", which the user, knowing the values used to randomize the message, can transform into a signature on the message. The actual 
blind signature is a WI proof of knowledge (PoK) of this signature, which prevents the signer from linking it to the signing session. This PoK is instantiated with Groth-Sahai (GS) commitments and proofs [GS08] for pairing-product equations (PPE), and the message space consists of pairs of group elements.

We require a lot more: the signer, without knowing the randomness used to hide the message, should not only make a commitment to a signature (which he cannot knowFootnote 10 on an unknown message, but in addition give a proof that this signature is valid. While the described blind signature scheme has the nice property that during issuing the user obtains an actual signature on the message, we show that its true potential has not yet been exploited. We first observe that the values the user sends to the signer for a blind signature can be seen as a commitment to the message. We then show that they actually suffice for the signer to directly - without the help of the user-construct a proof of knowledge of a signature on the message.

This is made possible by the specific structure of the signature, the fact that the commitments are homomorphic and a series of properties of the proof system. We prove that, besides being randomizable, Groth-Sahai proofs are homomorphid 3 w.r.t. the statement they prove (the product of two proofs is a proof for the product of the equations they prove), they are independent of parts of the statement-in some cases even of the committed value - , and there are ways to "blindly" transform a proof for one statement into a proof for another statement.

Instantiating Delegatable Anonymous Credentials. Belenkiy et al. [ $\left.\mathrm{BCC}^{+} 09\right]$ show that Groth-Sahai (GS) proofs can be randomized and combine them with an authentication scheme for secret keys to construct delegatable credentials. A pseudonym Nym is a commitment to the user's secret key and a credential is a proof of knowledge of an authentication chain. To issue or delegate, the issuer and the user jointly compute a PoK of an authenticator on the content of the user's pseudonym. In the case of delegation, the issuer prepends her own credential, after randomizing it. Their authentication scheme must satisfy strong security notions ( $F$-unforgeability and certification security), since secret keys cannot be extracted from the commitments, and an adversary against it must be allowed to ask for authenticators on as well as under the attacked key.

We avoid these notions and interactivity of delegation by following a more modular approach replacing the authenticators on secret keys by commuting signatures on verification keys. The underlying signatures are automorphic [Fuc09], which means that they are Groth-Sahai compatible and their verification keys lie in the message spacewhich is a requirement for delegation. A credential is then a chain of verification keys and certificates (as in the non-anonymous case), which are all given as commitments completed with proofs of validity.

Commuting signatures enable non-interactive issuing and delegation: given a user's pseudonym $N_{y} m_{U}$ (i.e., a commitment to his verification key), the issuer can produce a commitment $\mathbf{c}_{\Sigma}$ to a signature on the value committed in $N y m_{U}$ and a proof $\pi$ of validity using SigCom. In the case of issuing, the credential is $\left(\mathbf{c}_{\Sigma}, \pi\right)$ and is verified by checking $\pi$ on the issuer's public key, $N y m_{U}$ and $\mathbf{c}_{\Sigma}$. In the case of delegation, the issuer also randomizes her own credential cred $_{I}$, yielding a credential cred $_{I}{ }^{\prime}$ on her pseudonym $\mathrm{Nym}_{I}$ that is unlinkable to $\operatorname{cred}_{I}$. Running $\mathrm{AdC}_{\mathcal{K}}$, the issuer adapts the proof $\pi$ (which

\footnotetext{
${ }^{3}$ For linear equations the homomorphic property of GS proofs was also noted in [DHLW10].
} 
is valid under her verification key) to a proof $\widehat{\pi}$ of validity of the signature contained in $\mathbf{c}_{\Sigma}$ on the content of the pseudonym $N_{y} m_{U}$ under the content of the issuer's pseudonym $\operatorname{Nym}_{I}$. The credential for the user is then $\operatorname{cred}_{I}{ }^{\prime}\left\|N y m_{I}\right\|\left(\mathbf{c}_{\Sigma}, \widehat{\pi}\right)$.

Comparing Our Results to Previous Ones. Replacing the authenticators from the BCCKLS scheme with our automorphic signatures already more than doubles the efficiency. In the full version [Fuc10] we revise the approach to achieving simulatability of credentials. Groth and Sahai show how to simulate proofs of satisfiability of equations, consisting of commitments and proofs for the committed values, which are produced by the simulator. However, in order to simulate credentials for a given pseudonym, the simulator has to construct proofs for given commitments. Belenkiy et al. therefore double some of the commitments and provide proofs of consistency. We show that our credentials can be directly simulated even if some of the commitments are fixed beforehand.

Finally, our issuing (and delegation) protocol is significantly more efficient. While in $\left[\mathrm{BCC}^{+} 09\right]$, the issuer and the user run a complex two-party protocol using homomorphic encryption and interactive $\mathrm{ZK}$ proofs, in our instantiation the issuer simply sends a PoK of a signature. Both schemes are proven secure under the SXDH assumption and different "hidden" variants of the strong Diffie-Hellman assumption [BB04] (which are thus " $q$-type" assumptions): BB-CDH and BB-HSDH, introduced in $\left[\mathrm{BCC}^{+} 09\right]$, for their scheme and $\mathrm{ADH}-\mathrm{SDH}\left[\mathrm{AFG}^{+} 10\right]$ for ours (see Section 4.1).

Automorphic signatures were combined with GS proofs in $\mathrm{AFG}^{+} 10$ to construct anonymous proxy signatures (APS) [FP08]. They also allow one to prove rights in an anonymous way, but there is no anonymity between the delegator and the delegated user. If in our credential scheme we give the extraction key to a tracing authority, and define a proxy signing algorithm similar to delegation but outputting a committed signature on a clear message, we get an instantiation of APS with mutually anonymous delegation.

Subsequent to our work, Blazy et al. [BFPV11] defined a primitive similar to commuting signatures, called extractable signatures on randomizable ciphertexts. While their instantiation solely relies on the decision linear assumption (DLIN) [BBS04], it is only efficient for small message spaces due to bit-by-bit techniques.

\section{Preliminaries}

We briefly recall the definitions and security requirements for the relevant primitives from the literature (and refer to the full version [Fuc10] for more details).

Commitments. We will use a (non-interactive) randomizable extractable commitment scheme Com which is composed of the algorithms Setup, Com, RdCom, ExSetup, Extr, and WISetup. By $\mathcal{V}$ we denote the space of "committable" values, by $\mathcal{R}$ the randomness space and by $\mathcal{C}$ the space of commitments. On input the security parameter $1^{\lambda}$, Setup and WISetup output a commitment key $c k$, and ExSetup outputs (ck,ek), where $c k$ is distributed as the output of Setup; ek is called the extraction key. On input $c k$, a message $M \in \mathcal{V}$ and randomness $\rho \in \mathcal{R}$, Com outputs a commitment $\mathbf{c} \in \mathcal{C}$.

The scheme is perfectly binding, i.e., for any $c k \leftarrow$ Setup and any $\mathbf{c} \in \mathcal{C}$ there exists exactly one $M \in \mathcal{V}$ s.t. $\mathbf{c}=\operatorname{Com}(c k, M, \rho)$ for some $\rho$. If $(c k, e k) \leftarrow$ ExSetup then $\operatorname{Extr}(e k, \mathbf{c})$ extracts that value $M$ from c. The keys output by WISetup are computationally indistinguishable from those output by Setup and generate perfectly hiding 
commitments: for any $c k^{*} \leftarrow$ WISetup, $\mathbf{c} \in \mathcal{C}$ and $M \in \mathcal{V}$, there exists a $\rho \in \mathcal{R}$ s.t. $\mathbf{c}=\operatorname{Com}\left(c k^{*}, M, \rho\right)$. Finally, we have $\operatorname{Rd} \operatorname{Com}\left(c k, \operatorname{Com}(c k, M, \rho), \rho^{\prime}\right)=\operatorname{Com}(c k, M$, $\left.\rho+\rho^{\prime}\right)$; thus RdCom randomizes commitments 4 .

Proofs for Committed Values. We define a proof system that allows one to prove that committed values satisfy an equation. The proofs are constructed from the committed values and the used randomness, and they are witness indistinguishable, which means they do not reveal which satisfying values were used. Given a proof for a set of commitments, the proof can be adapted to a randomization of the commitments without knowledge of the committed values.

A randomizable witness-indistinguishable proof system Proof for a commitment scheme Com for a class $\mathcal{E}$ of equations consists of the algorithms Prove, Verify and RdProof. On input $c k$, an equation $\mathrm{E} \in \mathcal{E}$, values $M_{1}, \ldots, M_{n} \in \mathcal{V}$ satisfying $\mathrm{E}$ and $\rho_{1}, \ldots, \rho_{n} \in \mathcal{R}$, Prove outputs a proof $\pi$ for the values $\operatorname{Com}\left(c k, M_{1}, \rho_{1}\right), \ldots$, $\operatorname{Com}\left(c k, M_{n}, \rho_{n}\right)$. On input $c k, \mathrm{E}, \mathbf{c}_{1}, \ldots, \mathbf{c}_{n}$ and $\pi$, Verify outputs 0 or 1 , indicating rejection or acceptance of $\pi$. Every proof generated for commitments to values satisfying an equation is accepted by Verify. Given $c k, \mathbf{c}_{1}, \ldots, \mathbf{c}_{n}$, a proof $\pi$ for $\left(\mathbf{c}_{1}, \ldots, \mathbf{c}_{n}\right)$ and $\mathrm{E}$, and $\rho_{1}^{\prime}, \ldots, \rho_{n}^{\prime} \in \mathcal{R}$, algorithm RdProof outputs a proof for the randomizations $\mathbf{c}_{i}^{\prime}:=\operatorname{RdCom}\left(c k, \mathbf{c}_{i}, \rho_{i}^{\prime}\right)$; in particular, $\operatorname{RdProof}\left(c k, \mathrm{E},\left(\mathbf{c}_{1}, \rho_{1}^{\prime}\right), \ldots,\left(\mathbf{c}_{n}, \rho_{n}^{\prime}\right), \pi\right)$ is distributed as $\operatorname{Prove}\left(c k, \mathrm{E},\left(M_{1}, \rho_{1}+\rho_{1}^{\prime}\right), \ldots,\left(M_{n}, \rho_{n}+\rho_{n}^{\prime}\right)\right)$.

Soundness states that if there is a valid proof for a set of commitments for $\mathrm{E} \in \mathcal{E}$ then Extr extracts a set of values satisfying E. Witness indistinguishability is defined as follows: if the commitment key is output by WISetup then a set of commitments and a valid proof for them for an equation E reveals nothing, in an information-theoretical sense, about the committed values, except that they satisfy E.

Signatures. A signature scheme Sig consists of the following algorithms: Setup $\mathrm{S}_{\mathrm{S}}$ takes as input the security parameter $1^{\lambda}$ and outputs parameters $p p$, which define a message

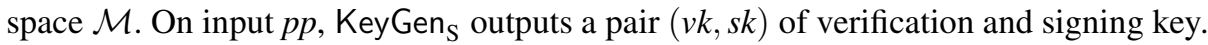
For $M \in \mathcal{M}, \operatorname{Sign}(s k, M)$ outputs a signature $\Sigma$, which is verified by $\operatorname{Ver}(v k, M, \Sigma)$. If $p p \leftarrow \operatorname{Setup}_{\mathrm{S}}$ and $(v k, s k) \leftarrow \operatorname{KeyGen}_{\mathrm{S}}(p p)$ then $\operatorname{Ver}(v k, M, \operatorname{Sign}(s k, M))=1$ for all $M \in \mathcal{M}$. Strong unforgeability means that given $v k$ and an oracle that queried on a message $M_{i}$ returns a signature $\Sigma_{i}$ on $M_{i}$, it is infeasible to output a pair $(M, \Sigma)$, s.t. $\operatorname{Ver}(v k, M, \Sigma)=1$ and $(M, \Sigma) \neq\left(M_{i}, \Sigma_{i}\right)$ for all $i$.

We require that Sig be compatible with Com and Proof: the messages, verification keys and signatures are composed of values in $\mathcal{V}$ (the value space of Com) and the signature verification predicate is a conjunction of equations from $\mathcal{E}$ (the class of equations for Proof). We note that from a compatible triple (Com, Proof, Sig) one can easily construct a verifiably encrypted signature scheme; see [Fuc10].

For our application to delegatable credentials we require furthermore that Sig be $a u$ tomorphic, that is, besides being compatible, its verification keys must lie in its message space $\mathcal{M}$. We let $\mathrm{E}_{\mathrm{Ver}}$ denote the verification equations for Sig. When, for example, $\Sigma$ is considered a variable we write $\mathrm{E}_{\operatorname{Ver}(v k, M, \cdot)}(\Sigma)$.

\footnotetext{
${ }^{4}$ Commitment schemes with two types of keys were called perfectly hiding with extraction in [GOS06] and strongly computationally hiding in [BCKL08]. Note that a scheme Com with the described properties is at the same time a lossy encryption scheme [BHY09].
} 


\section{Commuting Signatures and Verifiable Encryption}

Commuting signatures extend a commitment scheme Com, an associated proof system Proof and a compatible signature scheme Sig by the following functionalities: $\operatorname{Com}_{\mathcal{M}}$ is a commitment scheme with the same keys as Com and whose message space is that of Sig. SigCom takes a $\mathbf{C o m}_{\mathcal{M}}$ commitment and a signing key, and produces a commitment to a signature on the committed message and a proof of validity. SmSigCom simulates SigCom and is given a signature instead of the signing key. Moreover, the algorithms $\mathrm{AdC}$ and $\mathrm{AdD}$ adapt proofs when (de)committing to a signature (subscript $\mathcal{S}$ ), a message (subscript $\mathcal{M}$ ) or a verification key (subscript $\mathcal{K}$ ).

Definition 1. A system of commuting signatures and verifiable encryption consists of an extractable commitment scheme Com $=$ (Setup, Com, RdCom, ExSetup, Extr, WISetup) with value space $\mathcal{V}$ and randomness space $\mathcal{R}$, a randomizable WI proof system Proof $=$ (Prove, Verify, RdProof) for Com, a compatible signature scheme $\mathrm{Sig}=\left(\right.$ Setup $_{\mathrm{S}}$, KeyGen $_{\mathrm{S}}$, Sign, Ver $)$ and the following algorithms. We let $c k \leftarrow$ Setup, $p p_{\mathrm{S}} \leftarrow \operatorname{Setup}_{\mathrm{S}},(v k, s k) \leftarrow \operatorname{KeyGen}_{\mathrm{S}}\left(p p_{\mathrm{S}}\right), M \in \mathcal{M}, \mu \in \mathcal{R}_{\mathcal{M}}$ and $p p:=\left(c k, p p_{\mathrm{S}}\right)$.

Com $_{\mathcal{M}}$. On input pp, a message $M \in \mathcal{M}$ and $\mu \in \mathcal{R}_{\mathcal{M}}$, algorithm Com Cutputs $_{\mathcal{M}}$ a commitment $\mathbf{C}$ in $\mathcal{C}_{\mathcal{M}}$, the space of commitments. $\mathrm{RdCom}_{\mathcal{M}}$ takes inputs pp, $\mathbf{C}$ and $\mu^{\prime} \in \mathcal{R}_{\mathcal{M}}$ and outputs a randomized commitment $\mathbf{C}^{\prime}$. On input ek output by ExSetup, and $\mathbf{C}$, Extr $\operatorname{Mutputs}_{\mathcal{M}}$ the committed value $M$. We require $\operatorname{Com}_{\mathcal{M}}:=$ (Setup, $\operatorname{Com}_{\mathcal{M}}, \mathrm{RdCom}_{\mathcal{M}}$, ExSetup, Extr ${ }_{\mathcal{M}}$, WISetup) to be a commitment scheme as defined in Section 2 and to be compatible with Proof, i.e., Prove and RdProof accept inputs from $\mathcal{R}_{\mathcal{M}}$ and Verify accepts $\operatorname{Com}_{\mathcal{M}}$ commitments.

$\operatorname{AdC}_{\mathcal{S}}(p p, v k, \mathbf{C},(\Sigma, \rho), \bar{\pi})$. If $\operatorname{Verify}\left(c k, \mathrm{E}_{\operatorname{Ver}(v k, \cdot, \Sigma)}, \mathbf{C}, \bar{\pi}\right)=1$ then the algorithm outputs $\pi$ which is distributed as $\left[\operatorname{Prove}\left(c k, \mathrm{E}_{\operatorname{Ver}(v k, \cdot, \cdot)},(M, \mu),(\Sigma, \rho)\right)\right]$, where $M$ and $\mu$ are such that $\mathbf{C}=\operatorname{Com}_{\mathcal{M}}(p p, M, \mu)$.

$\operatorname{AdD}_{\mathcal{S}}(p p, v k, \mathbf{C},(\Sigma, \rho), \pi)$. If $\operatorname{Verify}\left(c k, \mathrm{E}_{\operatorname{Ver}(v k, \cdot, \cdot)}, \mathbf{C}, \operatorname{Com}(c k, \Sigma, \rho), \pi\right)=1$ then the algorithm outputs $\bar{\pi}$ which is distributed as $\left[\operatorname{Prove}\left(c k, \mathrm{E}_{\operatorname{Ver}(v k, \cdot, \Sigma)},(M, \mu)\right)\right]$, where $M$ and $\mu$ are such that $\mathbf{C}=\operatorname{Com}_{\mathcal{M}}(p p, M, \mu)$.

$\operatorname{AdC}_{\mathcal{M}}\left(p p, v k,(M, \mu), \mathbf{c}_{\Sigma}, \tilde{\pi}\right)$. If $\operatorname{Verify}\left(c k, \mathrm{E}_{\operatorname{Ver}(v k, M, \cdot)}, \mathbf{c}_{\Sigma}, \tilde{\pi}\right)=1$ then the algorithm outputs $\pi$ which is distributed as $\left[\operatorname{Prove}\left(c k, \mathrm{E}_{\operatorname{Ver}(v k, \cdot, \cdot)},(M, \mu),(\Sigma, \rho)\right)\right]$, where $\Sigma$ and $\rho$ are such that $\mathbf{c}_{\Sigma}=\operatorname{Com}(c k, \Sigma, \rho)$.

$\operatorname{AdD}_{\mathcal{M}}\left(p p, v k,(M, \mu), \mathbf{c}_{\Sigma}, \pi\right)$. If $\operatorname{Verify}\left(c k, \operatorname{EVer}_{\operatorname{lo}, \cdot, \cdot)}, \operatorname{Com}_{\mathcal{M}}(p p, M, \mu), \mathbf{c}_{\Sigma}, \pi\right)=1$, the algorithm outputs $\widetilde{\pi}$ which is distributed as $\left[\operatorname{Prove}\left(c k, \mathrm{E}_{\operatorname{Ver}(v k, M, \cdot)},(\Sigma, \rho)\right)\right]$, where $\Sigma$ and $\rho$ are such that $\mathbf{c}_{\Sigma}=\operatorname{Com}(c k, \Sigma, \rho)$.

$\operatorname{AdC}_{\mathcal{K}}\left(p p,(v k, \xi), \mathbf{C}, \mathbf{c}_{\Sigma}, \pi\right)$. If $\operatorname{Verify}\left(c k, \mathrm{E}_{\operatorname{Ver}(v k, \cdot, \cdot)}, \mathbf{C}, \mathbf{c}_{\Sigma}, \pi\right)=1$, the algorithm outputs $\widehat{\pi}$ which is distributed as $\left.\left[\operatorname{Prove}\left(c k, \mathrm{E}_{\operatorname{Ver}(\cdot, \cdot, \cdot)},(v k, \xi),(M, \mu),(\Sigma, \rho)\right)\right)\right]$, where $M, \mu, \Sigma$ and $\rho$ are such that $\mathbf{C}=\operatorname{Com}_{\mathcal{M}}(p p, M, \mu)$ and $\mathbf{c}_{\Sigma}=\operatorname{Com}(c k, \Sigma, \rho)$.

$\operatorname{AdD}_{\mathcal{K}}\left(p p,(v k, \xi), \mathbf{C}, \mathbf{c}_{\Sigma}, \widehat{\pi}\right)$. If $\operatorname{Verify}\left(c k, \operatorname{EVer}_{(\cdot, \cdot, \cdot)}, \operatorname{Com}(c k, v k, \xi), \mathbf{C}, \mathbf{c}_{\Sigma}, \widehat{\pi}\right)=1$, the algorithm outputs $\pi$, distributed as $\left.\left[\operatorname{Prove}\left(c k, \mathrm{E}_{\operatorname{Ver}(v k, \cdot, \cdot)},(M, \mu),(\Sigma, \rho)\right)\right)\right]$, where $M, \mu, \Sigma$ and $\rho$ are such that $\mathbf{C}=\operatorname{Com}_{\mathcal{M}}(p p, M, \mu)$ and $\mathbf{c}_{\Sigma}=\operatorname{Com}(c k, \Sigma, \rho)$. 
$\operatorname{Sig} \operatorname{Com}(p p, s k, \mathbf{C})$. If $\mathbf{C} \in \mathcal{C}_{\mathcal{M}}$ then the algorithm outputs a commitment to a signature and a proof of validity $\left(\mathbf{c}_{\Sigma}, \pi\right)$ which is distributed as

$$
\left[\Sigma \leftarrow \operatorname{Sign}(s k, M) ; \rho \leftarrow \mathcal{R}:\left(\operatorname{Com}(c k, \Sigma, \rho), \operatorname{Prove}\left(c k, \operatorname{Ever}_{\operatorname{Vek}, \cdot, \cdot)},(M, \mu),(\Sigma, \rho)\right)\right)\right]
$$

where $M$ and $\mu$ are such that $\mathbf{C}=\operatorname{Com}_{\mathcal{M}}(p p, M, \mu)$.

$\operatorname{SmSigCom}(p p, e k, v k, \mathbf{C}, \Sigma)$. Assume $(c k, e k) \leftarrow \operatorname{ExSetup.~If~} \operatorname{Ver}\left(v k, \operatorname{Extr}_{\mathcal{M}}(e k, \mathbf{C})\right.$,

$\Sigma)=1$ then the algorithm outputs $\left(\mathbf{c}_{\Sigma}, \pi\right)$ which is distributed as $[\rho \leftarrow \mathcal{R}$ :

$\left(\operatorname{Com}(c k, \Sigma, \rho)\right.$, Prove $\left.\left.\left(c k, \mathrm{E}_{\operatorname{Ver}(v k, \cdot, \cdot)},(M, \mu),(\Sigma, \rho)\right)\right)\right]$, where $M$ and $\mu$ are such that $\mathbf{C}=\operatorname{Com}_{\mathcal{M}}(p p, M, \mu)$.

By Algs $:=\left(\operatorname{AdC}_{\mathcal{S}}, \operatorname{AdD}_{\mathcal{S}}, \operatorname{AdC}_{\mathcal{M}}, \operatorname{AdD}_{\mathcal{M}}, \mathrm{AdC}_{\mathcal{K}}, \operatorname{AdD}_{\mathcal{K}}\right.$, SigCom, SmSigCom $)$ we denote the algorithms of the system that extend Com, Proof, Sig and $\mathbf{C o m}_{\mathcal{M}}$. When verifying a signature $\Sigma$ on a message $M$ by $\operatorname{Ver}(v k, M, \Sigma)$, we implicitly assume that Ver also checks whether $M \in \mathcal{M}$. Analogously, we assume that when verifying a proof of validity by running Verify on $E_{V e r}$ and $\mathbf{C}$, it checks whether $\mathbf{C} \in \mathcal{C}_{\mathcal{M}}$.

Definition 1 implies that running SigCom on a commitment to $M$ yields the same (the output is distributed identically) as running $\Sigma \leftarrow \operatorname{Sign}(s k, M), \operatorname{Com}_{\mathcal{M}}$ on $M$, Com on $\Sigma$ and Prove for $\mathrm{E}_{\operatorname{Ver}(v k, \cdot, \cdot)}$; or running Sign, $\operatorname{Com}_{\mathcal{M}}$ on $M$ and Prove for $\mathrm{E}_{\operatorname{Ver}(v k, \cdot, \Sigma)}$, and then Com on $\Sigma$ and $\operatorname{AdC}_{\mathcal{S}}$, etc. This means that the diagram in Figure 1 commutes.

SmSigCom allows us to prove the following unforgeability property: Consider an adversary that is given $(p p, e k, v k)$ for $(v k, s k) \leftarrow$ KeyGen $_{\mathrm{S}}$ and access to an oracle that on input $\mathbf{C}_{i}$ returns $\left(\mathbf{c}_{i}, \pi_{i}\right) \leftarrow \operatorname{Sig} \operatorname{Com}\left(p p, s k, \mathbf{C}_{i}\right)$. Then it cannot output a valid triple $\left(\mathbf{C}, \mathbf{c}_{\Sigma}, \pi\right)$ such that the committed message/signature pair is different from every pair committed in $\left(\mathbf{C}_{i}, \mathbf{c}_{i}\right)$ from the oracle calls. Commuting signatures moreover yield a round-optimal blind signature scheme: The user sends a commitment to the message to the signer, who runs SigCom to produce $\left(\mathbf{c}_{\Sigma}, \pi\right)$. The user computes a proof $\widetilde{\pi}$ for $\mathbf{c}_{\Sigma}$ and $M$ via $\operatorname{AdD}_{\mathcal{M}}$, and outputs $\left(\mathbf{c}_{\Sigma}, \tilde{\pi}\right)$ as the blind signature (see [Fuc10]).

\section{Instantiation of the Building Blocks}

\subsection{Bilinear Groups and Assumptions}

A bilinear group is a tuple $\mathcal{G}=\left(p, \mathbb{G}_{1}, \mathbb{G}_{2}, \mathbb{G}_{T}, e, G_{1}, G_{2}\right)$ where $\mathbb{G}_{1}, \mathbb{G}_{2}$ and $\mathbb{G}_{T}$ are cyclic groups of prime order $p ; G_{1}$ and $G_{2}$ generate $\mathbb{G}_{1}$ and $\mathbb{G}_{2}$, respectively; and $e: \mathbb{G}_{1} \times \mathbb{G}_{2} \rightarrow \mathbb{G}_{T}$ is an efficient non-degenerate bilinear map: $\forall X \in \mathbb{G}_{1} \forall Y \in \mathbb{G}_{2}$ $\forall a, b \in \mathbb{Z}: e\left(X^{a}, Y^{b}\right)=e(X, Y)^{a b}$, and $e\left(G_{1}, G_{2}\right)$ generates $\mathbb{G}_{T}$.

We denote group elements by capital letters and assume two fixed generators $G$ and $H$ of $\mathbb{G}_{1}$ and $\mathbb{G}_{2}$, respectively. We call a pair $(A, B) \in \mathbb{G}_{1} \times \mathbb{G}_{2}$ a Diffie-Hellman pair (w.r.t. $(G, H)$ ), if there exists $a \in \mathbb{Z}_{p}$ such that $A=G^{a}$ and $B=H^{a}$. We let $\mathcal{D H}:=\left\{\left(G^{a}, H^{a}\right) \mid a \in \mathbb{Z}_{p}\right\}$ denote the set of DH pairs. Using the bilinear map $e$, $\mathcal{D} \mathcal{H}$ is efficiently decidable by checking $e\left(G^{-1}, \underline{B}\right) e(\underline{A}, H)=1$. We will make the following assumptions.

Assumption 1 (SXDH). The Symmetric External Diffie-Hellman assumption for $\mathcal{G}$ states that given $\left(G_{1}, G_{1}^{r}, G_{1}^{s}, G_{1}^{t}\right)$ for random $r, s \in \mathbb{Z}_{p}$, it is hard to decide whether $t=r s$ or $t$ is random; likewise, given $\left(G_{2}, G_{2}^{r^{\prime}}, G_{2}^{s^{\prime}}, G_{2}^{t^{\prime}}\right)$ for random $r^{\prime}, s^{\prime} \in \mathbb{Z}_{p}$, it is hard to decide whether $t^{\prime}=r^{\prime} s^{\prime}$ or $t^{\prime}$ is random. 
The q-Asymmetric Double Hidden Strong Diffie-Hellman assumption (ADH-SDH) was introduced in $\left[\mathrm{AFG}^{+} 10\right]$ and proven to hold in the generic-group model for any type of pairing. It was shown in [FPV09] that under the $q$-SDH assumption [BB04], given $q-1$ tuples $\left(\left(K \cdot G^{v_{i}}\right)^{1 /\left(x+c_{i}\right)}, c_{i}, v_{i}\right)$ for random $c_{i}, v_{i} \leftarrow \mathbb{Z}_{p}$, it is hard to produce a new tuple of this form. Similarly to $q$-HSDH [BW07], ADH-SDH states that if $c_{i}$ and $v_{i}$ are given in a hidden form $\left(F^{c_{i}}, H^{c_{i}}, G^{v_{i}}, H^{v_{i}}\right)$, it is intractable to produce another such tuple $\left(\left(K \cdot G^{v}\right)^{1 /(x+c)}, F^{c}, H^{c}, G^{v}, H^{v}\right)$.

Assumption 2 ( $\boldsymbol{q}$-ADH-SDH). For randomly chosen $G, F, K \leftarrow \mathbb{G}_{1}, H \leftarrow \mathbb{G}_{2}$ and $x, c_{i}, v_{i} \leftarrow \mathbb{Z}_{p}$, given $\left(G, F, K, X=G^{x} ; H, Y=H^{x}\right)$ and, for $1 \leq i \leq q-1$,

$$
\left(A_{i}=\left(K \cdot G^{v_{i}}\right)^{\frac{1}{x+c_{i}}}, B_{i}=F^{c_{i}}, D_{i}=H^{c_{i}}, V_{i}=G^{v_{i}}, W_{i}=H^{v_{i}}\right),
$$

it is hard to output $\left(\left(K \cdot G^{v}\right)^{1 /(x+c)}, F^{c}, H^{c}, G^{v}, H^{v}\right)$ with $(c, v) \neq\left(c_{i}, v_{i}\right)$ for all $i$.

The next assumption is a weak variant of the various flexible $C D H$ assumptions, generalized to asymmetric bilinear groups. It was also introduced in [AFG $\left.{ }^{+} 10\right]$ and shown to be implied by DDH in $\mathbb{G}_{1}$, and thus by SXDH.

Assumption 3 (AWF-CDH). Given random generators $G \leftarrow \mathbb{G}_{1}$ and $H \leftarrow \mathbb{G}_{2}$, and $A=G^{a}$ for $a \leftarrow \mathbb{Z}_{p}$, it is hard to output $\left(G^{r}, G^{r a}, H^{r}, H^{r a}\right)$ with $r \neq 0$.

\subsection{Groth-Sahai Proofs and Automorphic Signatures}

Commitments. We instantiate Com, defined in Section 2, by the commitment scheme based on SXDH from [GS08]. Setup, on input $\mathcal{G}=\left(p, \mathbb{G}_{1}, \mathbb{G}_{2}, \mathbb{G}_{T}, e, G_{1}, G_{2}\right)$, outputs a commitment key $c k \in \mathbb{G}_{1}^{2 \times 2} \times \mathbb{G}_{2}^{2 \times 2}$. Value and randomness space are defined as $\mathcal{V}:=\mathbb{G}_{1} \cup \mathbb{G}_{2}$ and $\mathcal{R}:=\mathbb{Z}_{p}^{2}$. $\operatorname{Com}(c k, X, r)$ takes randomness $r \in \mathcal{R}$ and an element $X \in \mathcal{V}$; commitments to $\mathbb{G}_{1}$-elements are in $\mathbb{G}_{1}^{2}$ and commitments to $\mathbb{G}_{2}$-elements are in $\mathbb{G}_{2}^{2}$. We have $\operatorname{Com}(c k, X, r) \odot \operatorname{Com}\left(c k, X^{\prime}, r^{\prime}\right)=\operatorname{Com}\left(c k, X \cdot X^{\prime}, r+r^{\prime}\right)$, where " $\odot$ " denotes component-wise multiplication; the commitments are thus homomorphic.

$\operatorname{RdCom}\left(c k, \mathbf{c}, r^{\prime}\right)$ returns $\mathbf{c}^{\prime}:=\mathbf{c} \odot \operatorname{Com}\left(c k, 1, r^{\prime}\right)$, which for $\mathbf{c}=\operatorname{Com}(c k, X, r)$ is $\mathbf{c}^{\prime}=\operatorname{Com}\left(c k, X, r+r^{\prime}\right)$. ExSetup constructs $c k$ as in Setup and in addition outputs the used randomness as $e k$, and $\operatorname{Extr}(e k, \mathbf{c})$ outputs the committed value. WISetup produces a commitment key $c k^{*}$ that is indistinguishable from outputs of Setup under the SXDH assumption. Commitments under $c k^{*}$ are independent of the committed value.

Proofs for Committed Values. In order to instantiate Proof for Com, we use the proof system from [GS08], which was shown to be randomizable in [BCC $\left.{ }^{+} 09\right]$. The class of equations $\mathcal{E}$ for our proof system are pairing-product equations (PPE). A PPE over variables $X_{1}, \ldots, X_{m} \in \mathbb{G}_{1}$ and $Y_{1}, \ldots, Y_{n} \in \mathbb{G}_{2}$ is an equation of the form 5

$$
\begin{aligned}
& \mathrm{E}\left(X_{1}, \ldots, X_{m} ; Y_{1}, \ldots, Y_{n}\right): \\
& \prod_{j=1}^{n} e\left(A_{j}, \underline{Y_{j}}\right) \prod_{i=1}^{m} e\left(\underline{X_{i}}, B_{i}\right) \prod_{i=1}^{m} \prod_{j=1}^{n} e\left(\underline{X_{i}}, \underline{Y_{j}}\right)^{\gamma_{i, j}}=\mathbf{t}_{T},
\end{aligned}
$$

\footnotetext{
${ }^{5}$ For a more concise exposition we will underline the variables of an equation.
} 
defined by $A_{j} \in \mathbb{G}_{1}, B_{i} \in \mathbb{G}_{2}, \gamma_{i, j} \in \mathbb{Z}_{p}$, for $1 \leq i \leq m$ and $1 \leq j \leq n$, and $\mathbf{t}_{T} \in \mathbb{G}_{T}$. We refer to [GS08] or [Fuc10] for a description of the implementations of the following: Prove, which chooses internal randomness $Z \leftarrow \mathbb{Z}_{p}^{2 \times 2}$, and outputs $\pi \in \mathbb{G}_{2}^{2 \times 2} \times \mathbb{G}_{1}^{2 \times 2}$ (we write $\operatorname{Prove}\left(c k, \mathrm{E},\left(X_{i}, r_{i}\right)_{i=1}^{m},\left(Y_{j}, s_{j}\right)_{j=1}^{n} ; Z\right)$ if we want to make $Z$ explicit); $\operatorname{RdProof}\left(c k, \mathrm{E},\left(\mathbf{c}_{i}, r_{i}\right)_{i=1}^{m},\left(\mathbf{d}_{j}, s_{j}\right)_{j=1}^{n}, \pi\right)$, which adapts a proof $\pi$ to the new commitments output by $\operatorname{RdCom}\left(c k, \mathbf{c}_{i}, r_{i}\right)$ and $\operatorname{RdCom}\left(c k, \mathbf{d}_{j}, s_{j}\right)$; and $\operatorname{Verify}(c k, \mathrm{E}, \overrightarrow{\mathbf{c}}, \overrightarrow{\mathbf{d}}, \pi)$.

Signatures. We instantiate Sig with the automorphic signature scheme presented in $\left[\mathrm{AFG}^{+} 10\right.$. It is compatible, as signature components are in the space for committed values $\mathcal{V}=\mathbb{G}_{1} \cup \mathbb{G}_{2}$, and the verification equations are pairing-product equations, thus in $\mathcal{E}$. Moreover, the verification keys lie in its message space, the set of Diffie-Hellman pairs. Under $q-\mathrm{ADH}-\mathrm{SDH}$ and AWF-CDH (which is implied by SXDH), Sig is strongly unforgeable against adversaries making up to $q-1$ adaptive chosen-message queries, as shown in [Fuc09].

Scheme 1 (Sig). Setup has input a bilinear group $\mathcal{G}$ and outputs random generators $F, K, T \leftarrow \mathbb{G}_{1}$. The message space is $\mathcal{D} \mathcal{H}:=\left\{\left(G^{m}, H^{m}\right) \mid m \in \mathbb{Z}_{p}\right\}$.

KeyGen $_{\mathrm{S}}$ chooses $x \leftarrow \mathbb{Z}_{p}$ and outputs $v k=\left(G^{x}, H^{x}\right)$ and $s k=x$.

Sign has input a secret key $x$ and a message $(M, N) \in \mathcal{D H}$. It chooses $c, r \leftarrow \mathbb{Z}_{p}$ and outputs

$$
\left(A:=\left(K \cdot T^{r} \cdot M\right)^{\frac{1}{x+c}}, B:=F^{c}, D:=H^{c}, R:=G^{r}, S:=H^{r}\right) .
$$

Ver on input a verification key $(X, Y) \in \mathcal{D H}$, a message $(M, N) \in \mathcal{D} \mathcal{H}$ and a signature $(A, B, D, R, S)$ outputs 1 if and only if the following equalities hold:

$$
\begin{array}{ll}
e(A, Y \cdot D)=e(K \cdot M, H) e(T, S) & e(B, H)=e(F, D) \\
e(R, H)=e(G, S)
\end{array}
$$

Under SXDH and ADH-SDH, Com, Proof and Sig are instantiations of the primitives defined in Section 2. Note that if we based GS proofs on DLIN instead of SXDH, the security of our constructions would follow from DLIN, ADH-SDH and AWF-CDH, which can all be made for bilinear groups of every type (1, 2 and 3) from [GPS08].

\section{Additional Properties of Groth-Sahai Proofs}

We identify four properties of Groth-Sahai (GS) proofs which will allow us to instantiate commuting signatures. We refer to [Fuc10] for the proofs and further results. First, proofs are independent of the right-hand side of the equation, and if the equation does not contain pairings of two variables, i.e., $\gamma_{i j}=0$ for all $i, j$ in (1), then they are even independent of the committed values.

Lemma 1. For any equation $\mathrm{E} \in \mathcal{E}$ the output of $\operatorname{Prove}(\cdot, \mathrm{E}, \cdot, \cdot)$ is independent of $\mathbf{t}_{T}$.

Lemma 2. Proofs for equations for which $\gamma_{i j}=0$ for all $i, j$ depend only on the randomness of the commitments. 
Groth-Sahai (GS) proofs are homomorphic w.r.t. the equations, in that the product of two proofs is a proof for the "product of the respective equations". More precisely, given two equations

$$
\begin{aligned}
\mathrm{E} & : \prod_{i=1}^{n} e\left(A_{i}, \underline{Y_{i}}\right) \prod_{i=1}^{m} e\left(\underline{X_{i}}, B_{i}\right) \prod_{i=1}^{m} \prod_{j=1}^{n} e\left(\underline{X_{i}}, \underline{Y_{j}}\right)^{\gamma_{i, j}}=\mathbf{t}_{T} \\
\mathrm{E}^{\prime}: & \prod_{i=1}^{n^{\prime}} e\left(A_{i}^{\prime}, \underline{Y_{i}^{\prime}}\right) \prod_{i=1}^{m^{\prime}} e\left(\underline{X_{i}^{\prime}}, B_{i}^{\prime}\right) \prod_{i=1}^{m^{\prime}} \prod_{j=1}^{n^{\prime}} e\left(\underline{X_{i}^{\prime}}, \underline{Y_{j}^{\prime}}\right)^{\gamma_{i, j}^{\prime}}=\mathbf{t}_{T}^{\prime}
\end{aligned}
$$

and a proof $\pi$ for commitments $(\overrightarrow{\mathbf{c}}, \overrightarrow{\mathbf{d}})$ for E and a proof $\pi^{\prime}$ for commitments $\left(\overrightarrow{\mathbf{c}}^{\prime}, \overrightarrow{\mathbf{d}}^{\prime}\right)$ for $\mathrm{E}^{\prime}$, then $\pi^{\prime \prime}:=\pi \odot \pi^{\prime}$ is a proof for commitments $\left(\left(\overrightarrow{\mathbf{c}}, \overrightarrow{\mathbf{c}}^{\prime}\right),\left(\overrightarrow{\mathbf{d}}, \overrightarrow{\mathbf{d}}^{\prime}\right)\right)$ and equation $\mathrm{E}^{\prime \prime}$ defined as $\prod e\left(A_{i}, \underline{Y_{i}}\right) \prod e\left(A_{i}^{\prime}, \underline{Y_{i}^{\prime}}\right) \prod e\left(\underline{X_{i}}, B_{i}\right) \prod e\left(\underline{X_{i}^{\prime}}, B_{i}^{\prime}\right) \prod \prod e\left(\underline{X_{i}}, \underline{Y_{j}}\right)^{\gamma_{i, j}}$ $\cdot \prod \prod e\left(\underline{X_{i}^{\prime}}, Y_{j}^{\prime}\right)^{\gamma_{i, j}^{\prime}}=\mathbf{t}_{T}^{\prime \prime}$ (for arbitrary $\mathbf{t}_{T}^{\prime \prime} \in \mathbb{G}_{T}$ ).

Lemma 3. For $\mathrm{E}, \mathrm{E}^{\prime}$ and $\mathrm{E}^{\prime \prime}$ as above, if $\pi=\operatorname{Prove}\left(c k, \mathrm{E},\left(X_{i}, r_{i}\right)_{i=1}^{m},\left(Y_{j}, s_{j}\right)_{j=1}^{n} ; Z\right)$ and $\pi^{\prime}=\operatorname{Prove}\left(c k, \mathrm{E}^{\prime},\left(X_{i}^{\prime}, r_{i}^{\prime}\right)_{i=1}^{m^{\prime}},\left(Y_{j}^{\prime}, s_{j}^{\prime}\right)_{j=1}^{n^{\prime}} ; Z^{\prime}\right)$ then the following equation holds: $\pi \odot \pi^{\prime}=\operatorname{Prove}\left(c k, \mathrm{E}^{\prime \prime},\left(X_{i}, r_{i}\right)_{i=1}^{m},\left(X_{i}^{\prime}, r_{i}^{\prime}\right)_{i=1}^{m^{\prime}},\left(Y_{j}, s_{j}\right)_{j=1}^{n},\left(Y_{j}^{\prime}, s_{j}^{\prime}\right)_{j=1}^{n^{\prime}} ; Z+Z^{\prime}\right)$.

Given a proof for an equation, one can commit to its constants and adapt the proof. Consider $\mathrm{E}\left(X_{1}, \ldots, X_{m} ; Y_{1}, \ldots, Y_{n}\right)$ as in (1) and a proof $\pi$ for $\mathrm{E}$ and commitments $\left(\mathbf{c}_{1}, \ldots, \mathbf{c}_{m} ; \mathbf{d}_{1}, \ldots, \mathbf{d}_{n}\right)$. Then $\pi$ is also a proof for $\mathrm{E}^{\prime}\left(\vec{X}, A_{k} ; \vec{Y}\right)$ defined as

$$
\prod_{\substack{i \neq 1 \\ i \neq k}}^{n} e\left(A_{i}, \underline{Y_{i}}\right) \prod_{i=1}^{m} e\left(\underline{X_{i}}, B_{i}\right) \prod_{i=1}^{m} \prod_{j=1}^{n} e\left(\underline{X_{i}}, \underline{Y_{j}}\right)^{\gamma_{i, j}} e\left(\underline{A_{k}}, \underline{Y_{k}}\right)=\mathbf{t}_{T}
$$

and commitments $\left(\mathbf{c}_{1}, \ldots, \mathbf{c}_{m}, \operatorname{Com}\left(c k, A_{k}, 0\right) ; \mathbf{d}_{1}, \ldots, \mathbf{d}_{n}\right)$. We have thus:

Lemma 4. Let $\pi \leftarrow \operatorname{Prove}\left(c k, \mathrm{E},\left(X_{i}, r_{i}\right)_{i=1}^{m},\left(Y_{j}, s_{j}\right)_{j=1}^{n}\right), \mathbf{c}_{i}=\operatorname{Com}\left(c k, X_{i}, r_{i}\right)$ and $\mathbf{d}_{j}=\operatorname{Com}\left(c k, Y_{j}, s_{j}\right)$ for all $i, j$. Then $\operatorname{RdProof}\left(c k, \mathrm{E}^{\prime},\left(\mathbf{c}_{i}, 0\right)_{i=1}^{m},\left(\operatorname{Com}\left(c k, A_{k}, 0\right), r\right)\right.$, $\left.\left(\mathbf{d}_{j}, 0\right)_{j=1}^{n}, \pi\right)$ yields a proof that is distributed as Prove $\left(c k, \mathrm{E}^{\prime},\left(X_{i}, r_{i}\right)_{i=1}^{m},\left(A_{k}, r\right)\right.$, $\left.\left(Y_{j}, s_{j}\right)_{j=1}^{n}\right)$. An analogous result holds for committing to a constant $B_{k} \in \mathbb{G}_{2}$.

\section{Instantiation of Commuting Signatures}

We explain how to implement commuting signatures; due to space constraints we refer to [Fuc10] for more details and the proofs. In [Fuc09, $\mathrm{AFG}^{+} 10$ ], a blind signature scheme is constructed from the scheme Sig (Scheme1) as follows. Given parameters $(G, H, F, K, T)$ and a message $(M, N) \in \mathcal{D} \mathcal{H}:=\left\{\left(G^{m}, H^{m}\right) \mid m \in \mathbb{Z}_{p}\right\}$, the user chooses a random $t \leftarrow \mathbb{Z}_{p}$ and blinds the first message component by the factor $T^{t}$. He then sends the following to the signer: $U:=T^{t} \cdot M$, commitments $\mathbf{c}_{M}, \mathbf{c}_{N}, \mathbf{c}_{P}$ and $\mathbf{c}_{Q}$ to $M, N, P:=G^{t}$ and $Q:=H^{t}$, respectively; and proofs $\pi_{M}, \pi_{P}$ and $\pi_{U}$ proving $(M, N),(P, Q) \in \mathcal{D H}$ and well-formedness of $U$. With

$$
\begin{aligned}
\mathrm{E}_{\mathcal{D H}}(M, N) & : e\left(G^{-1}, \underline{N}\right) e(\underline{M}, H)=1 \\
\mathrm{E}_{U}(M, Q) & : e\left(T^{-1}, \underline{Q}\right) e\left(\underline{M}, H^{-1}\right)=e(U, H)^{-1}
\end{aligned}
$$

$\pi_{M}$ proves $\mathrm{E}_{\mathcal{D H}}(M, N), \pi_{P}$ proves $\mathrm{E}_{\mathcal{D H}}(P, Q)$, and $\pi_{U}$ proves $\mathrm{E}_{U}(M, Q)$, which asserts $U=T^{t} \cdot M$. 
The signer replies with a "pre-signature" on $U$, defined in (6) below, which the user converts into a signature and outputs a GS proof of knowledge of it. Now to turn this into a commuting signature, there are two key observations.

1. The values $\mathbf{C}:=\left(\mathbf{c}_{M}, \mathbf{c}_{N}, \pi_{M}, \mathbf{c}_{P}, \mathbf{c}_{Q}, \pi_{P}, U, \pi_{U}\right)$ the user sends to the signer can be considered as a commitment to the message $(M, N)$, which is extractable and randomizable, and which perfectly hides the message when the values are produced under a key $c k^{*} \leftarrow$ WISetup.

2. As Com is homomorphic, the values $\mathbf{c}_{P}$ and $\mathbf{c}_{Q}$ contained in the commitment $\mathbf{C}$ to $(M, N)$ can be used by the signer to compute commitments to an actual signature. Moreover, below we show how $\pi_{P}$ and $\pi_{U}$ can be used to make a proof of validity using Lemmas 1, 2, 3 and 4

For the blind signature scheme in [Fuc09], the values $\mathbf{c}_{P}, \mathbf{c}_{Q}, \pi_{P}$ and $\pi_{U}$ are mainly needed in the proof of unforgeability, when the simulator extracts the message, queries it to its signing oracle and then uses $P$ and $Q$ to turn the signature into a pre-signature. We show that these values can be directly used by the signer to produce commitments to the signature components and even a proof of validity.

Commitments to Messages. To instantiate $\operatorname{Com}_{\mathcal{M}}$, we define a commitment to a message $(M, N) \in \mathcal{D} \mathcal{H}$ as $\mathbf{C}$ discussed above. For parameters $p p=(\mathcal{G}, c k, F, K, T)$ the space of valid commitments is thus defined as

$$
\begin{array}{r}
\mathcal{C}_{\mathcal{M}}(p p):=\left\{\left(\mathbf{c}_{M}, \mathbf{c}_{N}, \pi_{M}, \mathbf{c}_{P}, \mathbf{c}_{Q}, \pi_{P}, U, \pi_{U}\right) \mid \operatorname{Verify}\left(c k, \mathrm{E}_{\mathcal{D H}}, \mathbf{c}_{M}, \mathbf{c}_{N}, \pi_{M}\right)\right. \\
\left.\wedge \operatorname{Verify}\left(c k, \mathrm{E}_{\mathcal{D H}}, \mathbf{c}_{P}, \mathbf{c}_{Q}, \pi_{P}\right) \wedge \operatorname{Verify}\left(c k, \mathrm{E}_{U}, \mathbf{c}_{M}, \mathbf{c}_{Q}, \pi_{U}\right)\right\},
\end{array}
$$

and the randomness space is $\mathcal{R}_{\mathcal{M}}:=\mathbb{Z}_{p} \times \mathcal{R}^{4}$. The algorithms Com $\operatorname{Cod}_{\mathcal{M}}, \operatorname{Rom}_{\mathcal{M}}$ and $\operatorname{Extr}_{\mathcal{M}}$ defining $\operatorname{Com}_{\mathcal{M}}$ are given in Figure 2]

Committing to a Signature on a Committed Message and Proving Validity. We now show how the signer can use the values in $\mathbf{C}$ to produce a proof of knowledge $\left(\mathbf{c}_{A}, \mathbf{c}_{B}, \mathbf{c}_{D}, \mathbf{c}_{R}, \mathbf{c}_{S}, \pi_{A}, \pi_{B}, \pi_{R}\right)$ of a signature $(A, B, D, R, S)$ (i.e., a verifiably encrypted signature) on the message committed in $\mathbf{C}$. The proofs $\pi_{A}, \pi_{B}$ and $\pi_{R}$ attest that the values committed in $\left(\mathbf{c}_{A}, \mathbf{c}_{B}, \mathbf{c}_{D}, \mathbf{c}_{R}, \mathbf{c}_{S}\right)$ and $\mathbf{c}_{M}$ contained in $\mathbf{C}$ satisfy the verification equations in (2):

$$
\begin{aligned}
\mathrm{E}_{A}(A, M ; S, D) & : e\left(T^{-1}, \underline{S}\right) e(\underline{A}, Y) e\left(\underline{M}, H^{-1}\right) e(\underline{A}, \underline{D})=e(K, H) \\
\mathrm{E}_{B}(B ; D) & : e\left(F^{-1}, \underline{D}\right) e(\underline{B}, H)=1 \\
\mathrm{E}_{R}(R ; S) & : e\left(G^{-1}, \underline{S}\right) e(\underline{R}, H)=1
\end{aligned}
$$

In the blind signature scheme, after receiving $\mathbf{C}$, the signer checks the proofs contained in it, and then produces a pre-signature, which is constructed as a signature on $U$, but on a message that lacks the second component: choose $c, r \leftarrow \mathbb{Z}_{p}$ and compute

$$
A:=\left(K \cdot T^{r} \cdot U\right)^{1 /(x+c)} \quad B:=F^{c} \quad D:=H^{c} \quad R^{\prime}:=G^{r} \quad S^{\prime}:=H^{r}
$$

Since $U:=T^{t} \cdot M$, we have $A=\left(K \cdot T^{r} \cdot U\right)^{1 /(x+c)}=\left(K \cdot T^{r+t} \cdot M\right)^{1 /(x+c)}$, which is the first component of a signature on $(M, N)$ with randomness $r+t$. Knowing $t$, 
Com $\mathcal{M}_{\mathcal{M}}$ on input $p p,(M, N) \in \mathcal{D} \mathcal{H},(t, \mu, \nu, \rho, \sigma) \in \mathcal{R}_{\mathcal{M}}$ sets $P=G^{t}, Q=H^{t}$ and returns

$$
\begin{aligned}
\mathbf{c}_{M}:=\operatorname{Com}(c k, M, \mu) & \mathbf{c}_{N}:=\operatorname{Com}(c k, N, \nu) & \pi_{M} \leftarrow \operatorname{Prove}\left(c k, \mathrm{E}_{\mathcal{D} \mathcal{H}},(M, \mu),(N, \nu)\right) \\
\mathbf{c}_{P}:=\operatorname{Com}(c k, P, \rho) & \mathbf{c}_{Q}:=\operatorname{Com}(c k, Q, \sigma) & \pi_{P} \leftarrow \operatorname{Prove}\left(c k, \mathrm{E}_{\mathcal{D} \mathcal{H}},(P, \rho),(Q, \sigma)\right) \\
U:=T^{t} \cdot M & & \pi_{U} \leftarrow \operatorname{Prove}\left(c k, \mathrm{E}_{U},(M, \mu),(Q, \sigma)\right)
\end{aligned}
$$

RdCom $\mathcal{M}$ has input $p p, \mathbf{C}$ and $\left(t^{\prime}, \mu^{\prime}, \nu^{\prime}, \rho^{\prime}, \sigma^{\prime}\right) \in \mathcal{R}_{\mathcal{M}}$, and returns $\mathbf{C}^{\prime}$. It replaces $t$ by $t+t^{\prime}$ setting $U^{\prime}:=U \cdot T^{t^{\prime}}, \hat{\mathbf{c}}_{P}:=\mathbf{c}_{P} \odot \operatorname{Com}\left(c k, G^{t^{\prime}}, 0\right)$, and $\hat{\mathbf{c}}_{Q}:=\mathbf{c}_{Q} \odot \operatorname{Com}\left(c k, H^{t^{\prime}}, 0\right)$. It then replaces the remaining randomness $(\mu, \nu, \rho, \sigma)$ by $\left(\mu+\mu^{\prime}, \nu+\nu^{\prime}, \rho+\rho^{\prime}, \sigma+\sigma^{\prime}\right)$, setting

$$
\begin{array}{rlrl}
\mathbf{c}_{M}^{\prime} & :=\operatorname{RdCom}\left(c k, \mathbf{c}_{M}, \mu^{\prime}\right) & \pi_{M}^{\prime} \leftarrow \operatorname{RdProof}\left(c k, \mathrm{E}_{\mathcal{D H}},\left(\mathbf{c}_{M}, \mu^{\prime}\right),\left(\mathbf{c}_{N}, \nu^{\prime}\right), \pi_{M}\right) \\
\mathbf{c}_{N}^{\prime}:=\operatorname{RdCom}\left(c k, \mathbf{c}_{N}, \nu^{\prime}\right) & & \\
\mathbf{c}_{P}^{\prime}:=\operatorname{RdCom}\left(c k, \hat{\mathbf{c}}_{P}, \rho^{\prime}\right) & \pi_{P}^{\prime} \leftarrow \operatorname{RdProof}\left(c k, \mathrm{E}_{\mathcal{D H}},\left(\hat{\mathbf{c}}_{P}, \rho^{\prime}\right),\left(\hat{\mathbf{c}}_{Q}, \sigma^{\prime}\right), \pi_{P}\right) \\
\mathbf{c}_{Q}^{\prime}:=\operatorname{RdCom}\left(c k, \hat{\mathbf{c}}_{Q}, \sigma^{\prime}\right) & \pi_{U}^{\prime} \leftarrow \operatorname{RdProof}\left(c k, \mathrm{E}_{U},\left(\mathbf{c}_{M}, \mu^{\prime}\right),\left(\hat{\mathbf{c}}_{Q}, \sigma^{\prime}\right), \pi_{U}\right)
\end{array}
$$

$\operatorname{Extr}_{\mathcal{M}}\left(e k, \mathbf{C}=\left(\mathbf{c}_{M}, \mathbf{c}_{N}, \pi_{M}, \mathbf{c}_{P}, \mathbf{c}_{Q}, \pi_{P}, U, \pi_{U}\right)\right)$ outputs $\left(\operatorname{Extr}\left(e k, \mathbf{c}_{M}\right), \operatorname{Extr}\left(e k, \mathbf{c}_{N}\right)\right)$.

$\operatorname{SigCom}(p p, s k, \mathbf{C})$. Parse $\mathbf{C}$ as $\left(\mathbf{c}_{M}, \mathbf{c}_{N}, \pi_{M}, \mathbf{c}_{P}, \mathbf{c}_{Q}, \pi_{P}, U, \pi_{U}\right)$ and $s k$ as $x$. If $\pi_{M}, \pi_{P}$ and $\pi_{U}$ are valid then choose $c, r \leftarrow \mathbb{Z}_{p}$ and $\alpha, \beta, \delta, \rho^{\prime}, \sigma^{\prime} \leftarrow \mathbb{Z}_{p}^{2}$ and compute the following values:

$$
\begin{array}{rlrl}
A & :=\left(K \cdot T^{r} \cdot U\right)^{\frac{1}{x+c}} & \mathbf{c}_{B}:=\operatorname{Com}\left(c k, F^{c}, \beta\right) & \mathbf{c}_{R}:=\mathbf{c}_{P} \odot \operatorname{Com}\left(c k, G^{r}, \rho^{\prime}\right) \\
\mathbf{c}_{A}:=\operatorname{Com}(c k, A, \alpha) & \mathbf{c}_{D}:=\operatorname{Com}\left(c k, H^{c}, \delta\right) & \mathbf{c}_{S}:=\mathbf{c}_{Q} \odot \operatorname{Com}\left(c k, H^{r}, \sigma^{\prime}\right) \\
\pi_{A}^{\prime}:=\pi_{U} \odot \operatorname{Prove}\left(c k, \mathrm{E}_{A^{\dagger}},(A, \alpha),\left(H^{c}, \delta\right) ; 0\right) & \quad\left(\text { with } \mathrm{E}_{A^{\dagger}} \text { being Equation 9) }\right) \\
\pi_{A} & \leftarrow \operatorname{RdProof}\left(c k, \mathrm{E}_{A},\left(\mathbf{c}_{A}, 0\right),\left(\mathbf{c}_{D}, 0\right),\left(\mathbf{c}_{M}, 0\right),\left(\mathbf{c}_{S}, \sigma^{\prime}\right), \pi_{A}^{\prime}\right) \\
\pi_{R} & \leftarrow \operatorname{RdProof}\left(c k, \mathrm{E}_{R},\left(\mathbf{c}_{R}, \rho^{\prime}\right),\left(\mathbf{c}_{S}, \sigma^{\prime}\right), \pi_{P}\right) & \pi_{B} \leftarrow \operatorname{Prove}\left(c k, \mathrm{E}_{\mathcal{D H}},\left(F^{c}, \beta\right),\left(H^{c}, \delta\right)\right)
\end{array}
$$

Return $\left(\mathbf{c}_{A}, \mathbf{c}_{B}, \mathbf{c}_{D}, \mathbf{c}_{R}, \mathbf{c}_{S}, \pi_{A}, \pi_{B}, \pi_{R}\right)$.

Fig. 2. Committing to messages and making commitments to a signature on a committed value

the user can fabricate an actual signature on $(M, N)$ from the pre-signature by setting $R:=R^{\prime} \cdot G^{t}=G^{r+t}$ and $S:=S^{\prime} \cdot H^{t}=H^{r+t} .(A, B, D, R, S)$ is then a signature on $(M, N)$ with randomness $(c, r+t)$.

Let $\mu, \rho$ and $\sigma$ denote the randomness of the respective commitments $\mathbf{c}_{M}, \mathbf{c}_{P}$ and $\mathbf{c}_{Q}$, contained in $\mathbf{C}$. Since the commitments are homomorphic, the signer can-without knowing $P=G^{t}$ and $Q=H^{t}$-compute commitments to $R$ and $S$ from $\mathbf{c}_{P}$ and $\mathbf{c}_{Q}$ :

$$
\begin{aligned}
& \mathbf{c}_{R}:=\operatorname{Com}\left(c k, R^{\prime}, 0\right) \odot \mathbf{c}_{P}=\operatorname{Com}(c k, R, \rho) \\
& \mathbf{c}_{S}:=\operatorname{Com}\left(c k, S^{\prime}, 0\right) \odot \mathbf{c}_{Q}=\operatorname{Com}(c k, S, \sigma)
\end{aligned}
$$

The signer then chooses $\alpha, \beta, \delta \leftarrow \mathcal{R}$, and makes the remaining commitments:

$$
\mathbf{c}_{A}:=\operatorname{Com}(c k, A, \alpha) \quad \mathbf{c}_{B}:=\operatorname{Com}(c k, B, \beta) \quad \mathbf{c}_{D}:=\operatorname{Com}(c k, D, \delta)
$$

The vector $\overrightarrow{\mathbf{c}}_{\Sigma}:=\left(\mathbf{c}_{A}, \mathbf{c}_{B}, \mathbf{c}_{D}, \mathbf{c}_{R}, \mathbf{c}_{S}\right)$ is thus a commitment to the signature $\Sigma=$ $(A, B, D, R, S)$ on $(M, N)$. It remains to construct proofs $\pi_{A}, \pi_{B}$ and $\pi_{R}$ for the 3 
equations in (5) - without knowledge of the randomness $\mu, \rho$ and $\sigma$ of the commitments $\mathbf{c}_{M}, \mathbf{c}_{R}$ and $\mathbf{c}_{S}$ ! This can be done by observing the following:

1. Equation $\mathrm{E}_{R}(R ; S)$ is actually $\mathrm{E}_{\mathcal{D H}}(R ; S)$ from (3). Since by (7) $\mathbf{c}_{R}$ and $\mathbf{c}_{P}$ have the same randomness $\rho$, and $\mathbf{c}_{S}$ and $\mathbf{c}_{Q}$ have the same randomness $\sigma$, and since by Lemma2 2 proofs for $\mathrm{E}_{\mathcal{D H}}$ are independent of the committed values, $\pi_{P}$ (the proof for $\mathbf{c}_{P}$ and $\mathbf{c}_{Q}$ for $\mathrm{E}_{\mathcal{D H}}$ ) is also a proof for $\mathbf{c}_{R}$ and $\mathbf{c}_{S}$; we thus set $\pi_{R}:=\pi_{P}$.

2. Lemmas 1 and 2 yield that proofs for $\mathrm{E}_{U}$ (Equation (4)) only depend on the randomness of the commitments. Since $\mathbf{c}_{S}=\operatorname{Com}(c k, S, \sigma)$ and $\mathbf{c}_{Q}=\operatorname{Com}(c k, Q, \sigma)$ have the same randomness, $\pi_{U}$ is not only a proof for $\mathrm{E}_{U}(M ; Q)$ but also for

$$
\mathrm{E}_{U^{\dagger}}(M ; S): e\left(T^{-1}, \underline{S}\right) e\left(\underline{M}, H^{-1}\right)=\mathbf{t}_{T}
$$

(for an arbitrary $\mathbf{t}_{T} \in \mathbb{G}_{T} \sqrt{6}$ for $\mathbf{c}_{M}$ and $\mathbf{c}_{S}$. Moreover, the signer, knowing $A, D$, $\alpha$ and $\delta$, can produce a proof $\pi_{A^{\dagger}} \leftarrow \operatorname{Prove}\left(c k, \mathrm{E}_{A^{\dagger}},(A, \alpha),(D, \delta)\right)$ for

$$
\mathrm{E}_{A^{\dagger}}(A ; D): e(\underline{A}, Y) e(\underline{A}, \underline{D})=\mathbf{t}_{T}
$$

(for any $\mathbf{t}_{T}$ ). Since the product of the left-hand sides of $\mathrm{E}_{U^{\dagger}}(M ; S)$ and $\mathrm{E}_{A^{\dagger}}(A ; D)$ is the left-hand side of $\mathrm{E}_{A}(A, M ; S, D)$ from (5), Lemma 3 yields that $\pi_{A}:=$ $\pi_{U} \odot \pi_{A^{\dagger}}$ is a proof for $\mathrm{E}_{A}$.

We have thus shown how the signer can construct $\pi_{A}$ and $\pi_{R}$. The remaining proof $\pi_{B}$ can be made regularly, since the required randomness $(\beta, \delta)$ was chosen by the signer. Finally, to get a random proof of knowledge, the signer randomizes all commitments and proofs using RdCom and RdProof as defined in Section 4.2. Algorithm SigCom, with some optimizations, is summarized in Figure 2.

Instantiation of SmSigCom. This algorithm is similar to SigCom but instead of the signing key $s k$ it is given a signature $(A, B, D, R, S)$ and the extraction key. It proceeds like SigCom but starting from a signature instead of producing a pre-signature: choose $\alpha, \beta, \delta, \rho^{\prime}, \sigma^{\prime} \leftarrow \mathcal{R}$ and set $\mathbf{c}_{A}, \mathbf{c}_{B}$ and $\mathbf{c}_{D}$ as in (8); use $e k$ to extract $P$ and $Q$ from $\mathbf{C}$ and set $\mathbf{c}_{R}:=\mathbf{c}_{P} \odot \operatorname{Com}\left(c k, R \cdot P^{-1}, \rho^{\prime}\right)=\operatorname{Com}\left(c k, R, \rho+\rho^{\prime}\right)$ and $\mathbf{c}_{S}:=\mathbf{c}_{Q} \odot \operatorname{Com}\left(c k, S \cdot Q^{-1}, \sigma^{\prime}\right)=\operatorname{Com}\left(c k, S, \sigma+\sigma^{\prime}\right)$. Now $\pi_{A}, \pi_{B}$ and $\pi_{R}$ can be computed as in SigCom in Figure2.

Instantiations of Proof Adaptation for Committing and Decommitting. We define equations $\mathrm{E}_{\widetilde{A}}$ and $\mathrm{E}_{\bar{A}}$ and recall $\mathrm{E}_{A}$, which all represent the first verification equation in (2) but with different elements being variables.

$$
\begin{aligned}
\mathrm{E}_{A}(A, M ; S, D) & : e\left(T^{-1}, \underline{S}\right) e(\underline{A}, Y) e\left(\underline{M}, H^{-1}\right) e(\underline{A}, \underline{D})=e(K, H) \\
\mathrm{E}_{\widetilde{A}}(A ; S, D) & : e\left(T^{-1}, \underline{S}\right) e(\underline{A}, Y) e(\underline{A}, \underline{D})=e(K \cdot M, H) \\
\mathrm{E}_{\bar{A}}(M) & : e\left(\underline{M}, H^{-1}\right)=e(A, Y \cdot D)^{-1} e(K, H) e(T, S)
\end{aligned}
$$

\footnotetext{
${ }^{6}$ Technically, $\pi_{U}$ is only a proof for $\mathrm{E}_{U^{\dagger}}$ when $\mathbf{t}_{T}$ is s.t. $M$ and $S$ satisfy it. However, since the proofs are independent of the right-hand side, the prover need not know the appropriate $\mathbf{t}_{T}$.
} 
With $\mathrm{E}_{B}$ and $\mathrm{E}_{R}$ defined in (5) we can write the following

$$
\begin{aligned}
& \mathrm{E}_{\operatorname{Ver}((X, Y), \cdot, \cdot)}((M, N),(A, B, D, R, S)) \\
& \quad \equiv \mathrm{E}_{A}(A, M ; S, D) \wedge \mathrm{E}_{B}(B ; D) \wedge \mathrm{E}_{R}(R ; S) \\
& \mathrm{E}_{\operatorname{Ver}((X, Y),(M, N), \cdot)}(A, B, D, R, S) \equiv \mathrm{E}_{\widetilde{A}}(A ; S, D) \wedge \mathrm{E}_{B}(B ; D) \wedge \mathrm{E}_{R}(R ; S) \\
& \quad \mathrm{E}_{\operatorname{Ver}((X, Y), \cdot,(A, B, D, R, S))}(M, N) \equiv \mathrm{E}_{\bar{A}}(M)
\end{aligned}
$$

$\mathrm{AdC}_{\mathcal{S}}$ transforms proofs $\bar{\pi}$ for $\mathrm{E}_{\operatorname{Ver}(v k, \cdot, \Sigma)}(12)$ into proofs $\pi$ for $\mathrm{E}_{\mathrm{Ver}(v k, \cdot, \cdot)}$ (10), $\mathrm{AdC}_{\mathcal{M}}$ transforms proofs $\tilde{\pi}$ for $\mathrm{E}_{\operatorname{Ver}(v k,(M, N), \cdot)} 11$ into proofs $\pi$ for $\mathrm{E}_{\operatorname{Ver}(v k, \cdot, \cdot)}$, whereas $\operatorname{AdD}_{\mathcal{S}}$ and $\mathrm{AdD}_{\mathcal{M}}$ do the converse.

Since the product of the left-hand sides of $\mathrm{E}_{\widetilde{A}}$ and $\mathrm{E}_{\bar{A}}$ is the left-hand side of $\mathrm{E}_{A}$, by Lemma 3 we have $\pi_{A}=\pi_{\widetilde{A}} \odot \pi_{\bar{A}}$, which lets us transform proofs for equations $\mathrm{E}_{\widetilde{A}}$ and $\mathrm{E}_{\bar{A}}$ into proofs for $\mathrm{E}_{A}$ and vice versa, and thus implement the four algorithms. Note that when a proof is multiplied by a freshly generated proof, it is uniformly distributed: by Lemma 3 , if $Z$ is the internal randomness of the proof and $Z^{\prime}$ that of the fresh proof then the randomness of the product of proofs is $Z+Z^{\prime}$. However, when reusing a proof it must be randomized first.

$\operatorname{AdC}_{\mathcal{S}}(p p, v k, \mathbf{C},((A, B, D, R, S),(\alpha, \beta, \delta, \rho, \sigma)), \bar{\pi})$. Proof $\bar{\pi}$ being for (12), it sets

$$
\pi_{\widetilde{A}} \leftarrow \operatorname{Prove}\left(c k, \mathrm{E}_{\widetilde{A}},(A, \alpha),(S, \sigma),(D, \delta)\right) \quad \begin{aligned}
& \pi_{B} \leftarrow \operatorname{Prove}\left(c k, \mathrm{E}_{B},(B, \beta),(D, \delta)\right) \\
& \pi_{R} \leftarrow \operatorname{Prove}\left(c k, \mathrm{E}_{R},(R, \rho),(S, \sigma)\right)
\end{aligned}
$$

for $\mathrm{E}_{B}$ and $\mathrm{E}_{R}$ defined in (5). It then returns $\pi:=\left(\pi_{\widetilde{A}} \odot \pi_{\bar{A}}, \pi_{B}, \pi_{R}\right)$.

$\operatorname{AdD}_{\mathcal{S}}(p p, v k, \mathbf{C},((A, B, D, R, S),(\alpha, \beta, \delta, \rho, \sigma)), \pi)$. The proof $\pi$ is of the form $\left(\pi_{A}\right.$, $\left.\pi_{B}, \pi_{R}\right)$. The algorithm sets $\pi_{\widetilde{A}} \leftarrow \operatorname{Prove}\left(c k, \mathrm{E}_{\widetilde{A}},(A, \alpha),(S, \sigma),(D, \delta)\right)$ and returns $\bar{\pi}:=\pi_{A} \oslash \pi_{\tilde{A}}$ (where " $\oslash$ " denotes componentwise division, i.e., multiplying every component of $\pi_{A}$ with the inverse of that component of $\pi_{\tilde{A}}$ ).

$\operatorname{AdC}_{\mathcal{M}}\left(p p, v k,((M, N),(t, \mu, \nu, \rho, \sigma)),\left(\mathbf{c}_{A}, \mathbf{c}_{B}, \mathbf{c}_{D}, \mathbf{c}_{R}, \mathbf{c}_{S}\right), \widetilde{\pi}\right)$. The proof $\tilde{\pi}$ is of the form $\left(\pi_{\widetilde{A}}, \pi_{B}, \pi_{R}\right)$. The algorithm returns $\pi:=\left(\pi_{\widetilde{A}} \odot \pi_{\bar{A}}, \pi_{B}^{\prime}, \pi_{R}^{\prime}\right)$ with

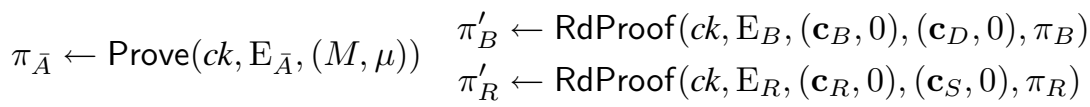

$\operatorname{AdD}_{\mathcal{M}}\left(p p, v k,((M, N),(t, \mu, \nu, \rho, \sigma)),\left(\mathbf{c}_{A}, \mathbf{c}_{B}, \mathbf{c}_{D}, \mathbf{c}_{R}, \mathbf{c}_{S}\right), \pi\right)$. The proof $\pi$ is of the form $\left(\pi_{A}, \pi_{B}, \pi_{R}\right)$. The algorithm computes $\pi_{\bar{A}}, \pi_{B}^{\prime}$ and $\pi_{R}^{\prime}$ as for $\operatorname{AdC}_{\mathcal{M}}$ above, and returns $\tilde{\pi}:=\left(\pi_{A} \oslash \pi_{\bar{A}}, \pi_{B}^{\prime}, \pi_{R}^{\prime}\right)$.

Instantiation of $\mathbf{A d C}_{\mathcal{K}}$ and $\mathbf{A d D}_{\mathcal{K}}$. A commitment $\mathbf{c}_{v k}$ to $v k=(X, Y) \in \mathcal{D} \mathcal{H}$ is defined as $\left(\operatorname{Com}(c k, X, \xi), \operatorname{Com}(c k, Y, \psi), \operatorname{Prove}\left(c k, \mathrm{E}_{\mathcal{D} \mathcal{H}},(X, \xi),(Y, \psi)\right)\right)$. The equations for $\mathrm{E}_{\operatorname{Ver}(\cdot, \cdot, \cdot)}((X, Y),(M, N),(A, B, D, R, S))$, i.e., when the key, the message and the signature are committed, are represented by

$$
\mathrm{E}_{\widehat{A}}(A, M ; S, Y, D): e\left(T^{-1}, \underline{S}\right) e\left(\underline{M}, H^{-1}\right) e(\underline{A}, \underline{Y}) e(\underline{A}, \underline{D})=e(K, H),
$$


and $\mathrm{E}_{B}$ and $\mathrm{E}_{R}$ from (5). Given a commitment $\mathbf{C}$ to a message, a commitment $\mathbf{c}_{\Sigma}=$ $\left(\mathbf{c}_{A}, \mathbf{c}_{B}, \mathbf{c}_{D}, \mathbf{c}_{R}, \mathbf{c}_{S}\right)$ to a signature, $(X, Y)$ and a proof $\pi=\left(\pi_{A}, \pi_{B}, \pi_{R}\right)$ of validity, by Lemma 4 the component $\pi_{A}$ can be adapted to $\pi_{\widehat{A}}$ for $\mathbf{c}_{Y}=\operatorname{Com}(c k, Y, \psi)$ setting

$$
\pi_{\widehat{A}} \leftarrow \operatorname{RdProof}\left(c k, \mathrm{E}_{\widehat{A}},\left(\mathbf{c}_{A}, 0\right),\left(\mathbf{c}_{M}, 0\right),\left(\mathbf{c}_{S}, 0\right),(\operatorname{Com}(c k, Y, 0), \psi),\left(\mathbf{c}_{D}, 0\right), \pi_{A}\right) .
$$

To adapt a proof to a decommitment of $\mathbf{c}_{v k}$, we have to reset the randomness of $\mathbf{c}_{Y}$ to $0 . \mathrm{AdD}_{\mathcal{K}}$ does thus the converse: it sets

$$
\pi_{A} \leftarrow \operatorname{RdProof}\left(c k, \mathrm{E}_{\widehat{A}},\left(\mathbf{c}_{A}, 0\right),\left(\mathbf{c}_{M}, 0\right),\left(\mathbf{c}_{S}, 0\right),(\operatorname{Com}(c k, Y, 0),-\psi),\left(\mathbf{c}_{D}, 0\right), \pi_{\widehat{A}}\right) .
$$

We conclude this section by summarizing the results by the following theorem.

Theorem 1. Under the ADH-SDH and the SXDH assumption, (Com, Proof, Sig, $\operatorname{Com}_{\mathcal{M}}, \mathrm{AdC}_{\mathcal{S}}, \mathrm{AdD}_{\mathcal{S}}, \mathrm{AdC}_{\mathcal{M}}, \mathrm{AdD}_{\mathcal{M}}, \mathrm{AdC}_{\mathcal{K}}, \mathrm{AdD}_{\mathcal{K}}$, SigCom, SmSigCom) is a system of commuting signatures and verifiable encryption as defined in Definition 1

\section{Non-interactively Delegatable Anonymous Credentials}

\subsection{The BCCKLS Model}

Functionality. We give an overview of the model for delegatable credentials defined in $\left[\mathrm{BCC}^{+} 09\right]$. The system parameters are set up by a trusted party. Every user generates a secret key $s k$, of which they can publish pseudonyms Nym. Any user can become originator of a credential by publishing a pseudonym $\mathrm{Nym}_{O}$ as the public key. To issue or delegate a credential, the issuer and the user (both known to each other under their respective pseudonyms) run a protocol at the end of which the user holds a credential. The holder can produce a credential proof for any of his pseudonyms, which proves that the owner of that pseudonym holds a credential rooted at a public key $\mathrm{Nym}_{O}$.

In our non-interactive instantiation we have the following: To delegate (or to issue) a credential to a user known to the delegator under $N y m_{U}$, the delegator produces (without interacting with the user) a ready credential proof for $N y m_{U}$. The user can turn this credential proof into a credential, which (as in the BCCKLS model) she can then use to make a credential proof for another pseudonym.

A (non-interactively) delegatable anonymous credential system consists of the following algorithms. On input $1^{\lambda}$, Setup $p_{\mathrm{C}}$ generates the parameters $p p$, which are input to all other algorithms. KeyGen ${ }_{C}$ generates user secret keys $s k$, of which NymGen outputs pseudonyms Nym and auxiliary information aux related to Nym.

Issuing and delegation is done via Issue, which on input the issuer's secret key $s k_{I}$, pseudonym $N y m_{I}$ and corresponding $\operatorname{aux}_{I}$, a level- $L$ credential cred for the issuer rooted at $\mathrm{Nym}_{O}$ (if $L=0$ then cred $=\varepsilon$ ) and a user pseudonym $\mathrm{Nym}_{U}$, outputs a credential proof credproof for $\mathrm{Nym}_{U}$. From this the user can obtain a credential cred by running Obtain on his secret key $s k_{U}$, pseudonym $N y m_{U}$ and aux the issuer's and delegator's pseudonyms $N y m_{O}$ and $N y m_{I}$, respectively, and credproof. Running CredProve on ( $p p, N_{y m_{O}}$, cred, sk, Nym, aux, L) permits a user to make a credential proof for the pseudonym Nym related to $s k$ and aux. CredVerify verifies a proof 
credproof rooted at $\mathrm{Nym}_{O}$ for $\mathrm{Nym}$. Note that CredProve outputs a credproof for the user that runs it, while Issue outputs a credproof for the user one issues or delegates to.

Security. Security is defined by correctness, anonymity and unforgeability. Run honestly, Issue and Obtain must produce credentials on which, for all user pseudonyms, CredProve outputs a proof that is accepted by CredVerify.

Anonymity means that an adversary interacting with honest users cannot distinguish the real game from an ideal game: There are simulated parameters which are indistinguishable from the real ones but lead to pseudonyms, credentials and proofs that are independent of users' secret keys. Given a trapdoor for these parameters, Issue, Obtain and CredProve can be simulated without the secret inputs cred, sk and aux.

To break unforgeability, an adversary must produce a proof that some Nym has a credential although such a credential has never been issued to any pseudonym of the owner of Nym. To formalize the notion of "owner", Belenkiy et al. define an algorithm that extracts from a pseudonym a user identity $v k$, which is uniquely defined by the secret key. Moreover, from a credential proof it extracts the identities that represent the underlying delegation chain. We say that a forgery occurs if the adversary produces a credential for authority $v k_{0}$ from which are extracted $\left(v k_{1}, \ldots, v k_{L-1}, v k_{L}\right)$ such that $v k_{L-1}$ is an honest user that never delegated a level- $L$ credential rooted at $v k_{0}$ to $v k_{L}$.

\subsection{Our Instantiation}

In the instantiation from $\left[\mathrm{BCC}^{+} 09\right]$ the system parameters are a Groth-Sahai (GS) commitment key and parameters for an authentication scheme. Each user holds a secret key $s k$ for the authentication scheme, and a pseudonym is a GS commitment to $f(s k)$ for a one-way function $f$. To issue and delegate, the issuer and the user run an interactive two-party protocol to compute a proof of knowledge of an authenticator on the user's secret key, which is valid under the issuer's secret key. A credential is then a chain of pseudonyms and committed authenticators with GS proofs of validity.

We replace the authenticators (consisting of 11 group elements and verified by 8 pairing-product equations) by automorphic signatures (5 group elements satisfying 3 PPEs). A non-anonymous level- $L$ credential for $v k_{L}$ rooted at $v k_{0}$ is a chain of verification keys and signatures $\left(\Sigma_{1}, v k_{1}, \Sigma_{2} \ldots, v k_{L-1}, \Sigma_{L}\right)$, where $\Sigma_{i}$ is a signature on $v k_{i}$ under $v k_{i-1}$. To achieve anonymity, the keys and signatures in the credential are committed to and proofs of validity are added. Using commuting signatures, given a commitment to a key, the issuer can directly make a commitment to a signature on it and a validity proof. This is what enables non-interactive delegation.

However, merely signing user keys does not suffice, as the issuer of a credential might want to add public information to the credential, such as attributes. For delegatable credentials it is also required to include the originator's pseudonym and the delegation level in each certificate to prevent combining different credentials and changing the order within a credential.

In the full version [Fuc10] we therefore give a simple extension of Sig: Scheme Sig $^{\prime \prime}$ has message space $\mathbb{Z}_{p} \times \mathcal{M}$, allowing the signer to specify a public value in addition to $M$. Its parameters contain one additional group element, but the signatures have the same size as those of Sig. We also define SigCom", an adaptation of SigCom which 
has the public value in $\mathbb{Z}_{p}$ as additional input, and show that all the other algorithms defined in Definition 1 and instantiated in Section 6 work equally for Sig and Sig $\mathbf{~}^{\prime 7}$.

Our Scheme. Let $\mathcal{H}: \mathcal{C}_{\mathcal{M}} \times \mathbb{N} \rightarrow \mathbb{Z}_{p}$ be a collision-resistant hash function. Then our scheme Cred can be sketched as follows: Setup ${ }_{C}$ generates a key for Com and parameters for $\mathbf{S i g}^{\prime \prime}$; KeyGen ${ }_{\mathrm{C}}$ outputs a signing key for $\mathbf{S i g}^{\prime \prime}$; and given such a key, NymGen outputs a commitment to the corresponding verification key and the used randomness as auxiliary information. A level- $L$ credential proof from $N y m_{0}$ for $N y m_{L}$ has the form

$$
\text { credproof }=\left(\mathbf{c}_{1}, \pi_{1}, \text { Nym }_{1}, \mathbf{c}_{2}, \pi_{2}, \ldots, N y m_{L-1}, \mathbf{c}_{L}, \pi_{L}\right)
$$

where $\mathbf{c}_{i}$ is a commitment to a $\mathbf{S i g}{ }^{\prime \prime}$ signature $\Sigma_{i}$ on the public value $\mathcal{H}\left(\mathrm{Nym}_{0}, i\right)$ and the key committed in $N y m_{i}$, valid under the key committed in $N y m_{i-1}$; and $\pi_{i}$ is a proof of validity of $\Sigma_{i}$. We call credproof a credential if it is valid on a "trivial" $N y m_{L}$, i.e., when $\operatorname{Nym}_{L}=\operatorname{Com}\left(c k, v k_{L}, 0\right)$.

CredProve takes a credential and turns it into a credential proof for $\mathrm{Nym}_{L}$ by randomizing all its components, using as randomness for the last component the value aux s.t. $\operatorname{Nym}_{L}=\operatorname{Com}\left(c k, v k_{L}, a u x\right)$. CredVerify verifies a credproof by checking the proofs contained in it. Given a level- $L$ credential, Issue extends it by one level making a credential proof for the delegatee's pseudonym $N_{y m_{L+1}}$ : In case of a delegation $(L>0)$, it first makes a credproof for the issuer's pseudonym $N y m_{I}$; otherwise credproof $:=\varepsilon$. Running SigCom" on $s k_{I}, \mathcal{H}\left(N_{y m_{0}}, L+1\right)$ and $N y m_{L+1}$, it produces $\left(\mathbf{c}_{L+1}, \pi_{L+1}^{\prime}\right)$, a verifiably encrypted signature under $v k_{I}$. It then runs $\mathrm{AdC}_{\mathcal{K}}$ on $\left(v k_{I}, a u x_{I}\right)$ to adapt $\pi_{L+1}^{\prime}$ to a proof $\pi_{L+1}$, which is valid for the committed verification key $N y m_{I}$. Finally, Issue outputs credproof $\left\|N y m_{I}\right\|\left(\mathbf{c}_{L+1}, \pi_{L+1}\right)$. Obtain turns a level- $L$ credential proof into a credential by adapting the randomness to make it valid for a trivial $\mathrm{Nym}_{L}$.

Simulatability. While unforgeability of our scheme is implied by the soundness of Proof and unforgeability of Sig", anonymity is shown as follows. We generate the simulated parameters by replacing Setup with WISetup; this makes the commitments perfectly hiding and thus pseudonyms and credential proofs independent of secret keys.

We then have to simulate issuing and CredProve for pseudonyms of the adversary's choice. This essentially means to simulate credential proofs for given Nym's; thus, simulating commitments to a signature and a proof of validity w.r.t. commitments to a key and to a message which are both given to the simulator.

While Groth and Sahai [GS08] show simulation of proofs of satisfiability of equations, where the simulator produces all the commitments, we require a novel type of simulation: given commitments to certain variables of an equation, we have to simulate the remaining commitments and the proof of validity. In the full version [Fuc10] we show that this can be done for a class $\mathcal{E}^{\prime} \subset \mathcal{E}$ of equations, in which the equations for validity of committed signatures fall. We call a commuting signature scheme simulatable if given a commitment to a key and a commitment to a message the simulator can create a verifiably encrypted signature for them.

In [Fuc10] we define a simulatable variant of our scheme from Section 6 . We also give a formal description of our credential scheme Cred and a proof of the following.

\footnotetext{
${ }^{7}$ Note that replacing SigCom by SigCom" in the construction of a blind signature at the end of Section 3 yields a partially blind signature [AF96].
} 
Theorem 2. Let (Com, Proof, $\left.\mathbf{S i g}^{\prime \prime}, \mathbf{C o m}_{\mathcal{M}}, \mathbf{A l g s}\right)$ be a system of commuting signatures which is automorphic and simulatable, and let $\mathcal{H}$ be a collision-resistant hash function. Then Cred is a secure delegatable anonymous credential scheme.

\section{Conclusion}

We introduced and instantiated commuting signatures and verifiable encryption. Given an encryption $\mathbf{C}$ of $M$ and a signing key, they let us produce an encryption $\mathbf{c}_{\Sigma}$ of a signature on $M$ and a proof that $\mathbf{c}_{\Sigma}$ contains a valid signature on the content of $\mathbf{C}$. We used them to give the first instantiation of delegatable anonymous credentials with non-interactive issuing and delegation and believe that they are a useful tool in the construction of privacy-preserving primitives that will find further applications.

\section{Acknowledgements}

The author would like to thank David Pointcheval, Elizabeth Quaglia and Damien Vergnaud for many helpful discussions and the anonymous referees of CCS 2010 and EUROCRYPT for their valuable comments.

\section{References}

[AF96] Abe, M., Fujisaki, E.: How to date blind signatures. In: Kim, K., Matsumoto, T. (eds.) ASIACRYPT 1996. LNCS, vol. 1163, pp. 244-251. Springer, Heidelberg (1996)

[AFG ${ }^{+}$10] Abe, M., Fuchsbauer, G., Groth, J., Haralambiev, K., Ohkubo, M.: StructurePreserving Signatures and Commitments to Group Elements. In: Rabin, T. (ed.) CRYPTO 2010. LNCS, vol. 6223, pp. 209-236. Springer, Heidelberg (2010)

[BB04] Boneh, D., Boyen, X.: Short signatures without random oracles. In: Cachin, C., Camenisch, J. (eds.) EUROCRYPT 2004. LNCS, vol. 3027, pp. 56-73. Springer, Heidelberg (2004)

[BBS04] Boneh, D., Boyen, X., Shacham, H.: Short group signatures. In: Franklin, M. (ed.) CRYPTO 2004. LNCS, vol. 3152, pp. 41-55. Springer, Heidelberg (2004)

$\left[\mathrm{BCC}^{+}\right.$09] Belenkiy, M., Camenisch, J., Chase, M., Kohlweiss, M., Lysyanskaya, A., Shacham, H.: Randomizable proofs and delegatable anonymous credentials. In: Halevi, S. (ed.) CRYPTO 2009. LNCS, vol. 5677, pp. 108-125. Springer, Heidelberg (2009)

[BCKL08] Belenkiy, M., Chase, M., Kohlweiss, M., Lysyanskaya, A.: P-signatures and noninteractive anonymous credentials. In: Canetti, R. (ed.) TCC 2008. LNCS, vol. 4948, pp. 356-374. Springer, Heidelberg (2008)

[BFM88] Blum, M., Feldman, P., Micali, S.: Non-interactive zero-knowledge and its applications. In: STOC, pp. 103-112. ACM Press, New York (1988)

[BFPV11] Blazy, O., Fuchsbauer, G., Pointcheval, D., Vergnaud, D.: Signatures on randomizable ciphertexts. In: Gennaro, R. (ed.) PKC 2011. LNCS, vol. 6571, pp. 403-422. Springer, Heidelberg (2011)

[BGLS03] Boneh, D., Gentry, C., Lynn, B., Shacham, H.: Aggregate and verifiably encrypted signatures from bilinear maps. In: Biham, E. (ed.) EUROCRYPT 2003. LNCS, vol. 2656, pp. 416-432. Springer, Heidelberg (2003) 
[BHY09] Bellare, M., Hofheinz, D., Yilek, S.: Possibility and impossibility results for encryption and commitment secure under selective opening. In: Joux, A. (ed.) EUROCRYPT 2009. LNCS, vol. 5479, pp. 1-35. Springer, Heidelberg (2009)

[Bra99] Brands, S.: Rethinking public key infrastructure and digital certificates-building privacy. PhD thesis, Eindhoven Inst. of Tech., The Netherlands (1999)

[BW07] Boyen, X., Waters, B.: Full-domain subgroup hiding and constant-size group signatures. In: Okamoto, T., Wang, X. (eds.) PKC 2007. LNCS, vol. 4450, pp. 1-15. Springer, Heidelberg (2007)

[Cha83] Chaum, D.: Blind signatures for untraceable payments. In: Chaum, D., Rivest, R.L., Sherman, A.T. (eds.) CRYPTO 1982, pp. 199-203. Plenum Press, New York (1983)

[Cha85] Chaum, D.: Security without identification: Transaction systems to make big brother obsolete. Commun. ACM 28(10), 1030-1044 (1985)

[CL01] Camenisch, J., Lysyanskaya, A.: An efficient system for non-transferable anonymous credentials with optional anonymity revocation. In: Pfitzmann, B. (ed.) EUROCRYPT 2001. LNCS, vol. 2045, pp. 93-118. Springer, Heidelberg (2001)

[CL02] Camenisch, J., Lysyanskaya, A.: A signature scheme with efficient protocols. In: Cimato, S., Galdi, C., Persiano, G. (eds.) SCN 2002. LNCS, vol. 2576, pp. 268289. Springer, Heidelberg (2003)

[CL04] Camenisch, J., Lysyanskaya, A.: Signature schemes and anonymous credentials from bilinear maps. In: Franklin, M. (ed.) CRYPTO 2004. LNCS, vol. 3152, pp. 56-72. Springer, Heidelberg (2004)

[CL06] Chase, M., Lysyanskaya, A.: On signatures of knowledge. In: Dwork, C. (ed.) CRYPTO 2006. LNCS, vol. 4117, pp. 78-96. Springer, Heidelberg (2006)

[Dam90] Damgård, I.: Payment systems and credential mechanisms with provable security against abuse by individuals. In: Goldwasser, S. (ed.) CRYPTO 1988. LNCS, vol. 403, pp. 328-335. Springer, Heidelberg (1990)

[DHLW10] Dodis, Y., Haralambiev, K., López-Alt, A., Wichs, D.: Cryptography against continuous memory attacks. In: FOCS, pp. 511-520. IEEE Computer Society, Los Alamitos (2010)

[Fis06] Fischlin, M.: Round-optimal composable blind signatures in the common reference string model. In: Dwork, C. (ed.) CRYPTO 2006. LNCS, vol. 4117, pp. 60-77. Springer, Heidelberg (2006)

[FP08] Fuchsbauer, G., Pointcheval, D.: Anonymous proxy signatures. In: Ostrovsky, R., De Prisco, R., Visconti, I. (eds.) SCN 2008. LNCS, vol. 5229, pp. 201-217. Springer, Heidelberg (2008)

[FPV09] Fuchsbauer, G., Pointcheval, D., Vergnaud, D.: Transferable constant-size fair Ecash. In: Garay, J.A., Miyaji, A., Otsuka, A. (eds.) CANS 2009. LNCS, vol. 5888, pp. 226-247. Springer, Heidelberg (2009)

[Fuc09] Fuchsbauer, G.: Automorphic signatures in bilinear groups and an application to round-optimal blind signatures. Cryptology ePrint Archive, Report 2009/320 (2009), http://eprint.iacr.org/2009/320 an extended abstract appeared as part of $\left[\mathrm{AFG}^{+} 10\right]$

[Fuc10] Fuchsbauer, G.: Commuting signatures and verifiable encryption and an application to non-interactively delegatable credentials. Cryptology ePrint Archive, Report 2010/233 (2010), http: / / eprint.iacr.org/2010/233

[GOS06] Groth, J., Ostrovsky, R., Sahai, A.: Perfect non-interactive zero knowledge for NP. In: Vaudenay, S. (ed.) EUROCRYPT 2006. LNCS, vol. 4004, pp. 339-358. Springer, Heidelberg (2006)

[GPS08] Galbraith, S.D., Paterson, K.G., Smart, N.P.: Pairings for cryptographers. Discrete Applied Mathematics 156(16), 3113-3121 (2008) 
[GS08] Groth, J., Sahai, A.: Efficient non-interactive proof systems for bilinear groups. In: Smart, N.P. (ed.) EUROCRYPT 2008. LNCS, vol. 4965, pp. 415-432. Springer, Heidelberg (2008)

[LRSW00] Lysyanskaya, A., Rivest, R.L., Sahai, A., Wolf, S.: Pseudonym systems. In: Heys, H.M., Adams, C.M. (eds.) SAC 1999. LNCS, vol. 1758, pp. 184-199. Springer, Heidelberg (2000)

[PS96] Pointcheval, D., Stern, J.: Provably secure blind signature schemes. In: Kim, K., Matsumoto, T. (eds.) ASIACRYPT 1996. LNCS, vol. 1163, pp. 252-265. Springer, Heidelberg (1996)

[RS09] Rückert, M., Schröder, D.: Security of verifiably encrypted signatures and a construction without random oracles. In: Shacham, H., Waters, B. (eds.) Pairing 2009. LNCS, vol. 5671, pp. 17-34. Springer, Heidelberg (2009) 